

\section{DISCLAIMER}

This report was prepared as an account of work sponsored by an agency of the United States Government. Neither the United States Government nor any agency Thereof, nor any of their employees, makes any warranty, express or implied, or assumes any legal liability or responsibility for the accuracy, completeness, or usefulness of any information, apparatus, product, or process disclosed, or represents that its use would not infringe privately owned rights. Reference herein to any specific commercial product, process, or service by trade name, trademark, manufacturer, or otherwise does not necessarily constitute or imply its endorsement, recommendation, or favoring by the United States Government or any agency thereof. The views and opinions of authors expressed herein do not necessarily state or reflect those of the United States Government or any agency thereof. 


\section{DISCLAIMER}

Portions of this document may be illegible in electronic image products. Images are produced from the best available original document. 
Operated by

Westinghouse

Hanford Company

for the USDOE

Contract No.

EY.76-C-14.2170
A Subsidiary of

Westinghouse Electric

Corporation

\section{Hanford Engineering Development Laboratory}

\section{INFORMATION CONCERNING USE OF THIS DOCUMENT}

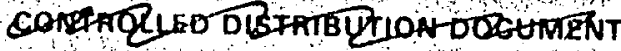

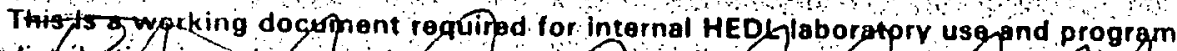
dis fripution in order to perform drect gr integrato mo wark of the BAp/FFA. 3 pogram distribytion s limited to HEDL OOE afo OOE contactors who roguro

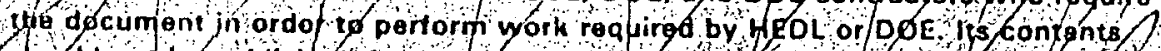

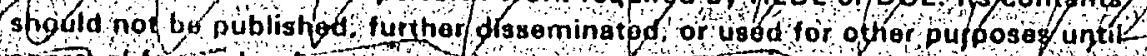

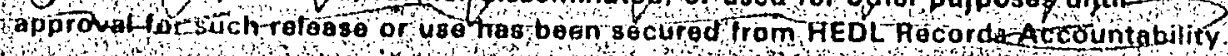
Notice

This documiont was prepared as an eccount of work sponsored by the United States Gavernment Neither tho United States nor tho United States Dopert ment of Energy, nor any of thel employues nor anyotitioli contractors subcontracto s, or their omployeas, makes anv warranty exprese or impllad or assumes any logal jability or responsibility far the accuracy completereso or usefulnese of any informatlon. opperatus, productor proces dloclosed or represents that it use would not Infringe prlvetely ownod rights. 


\title{
AS-BUILT DESCRIPTION OF THE EBR-II, RUN 97 DOSIMETRY EXPERIMENT
}

\section{Hanford Engineering Development Laboratory}

\author{
C. L. Long \\ J. A. Ulseth \\ E. P. Lippincott
}

\section{December 1978}

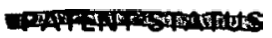

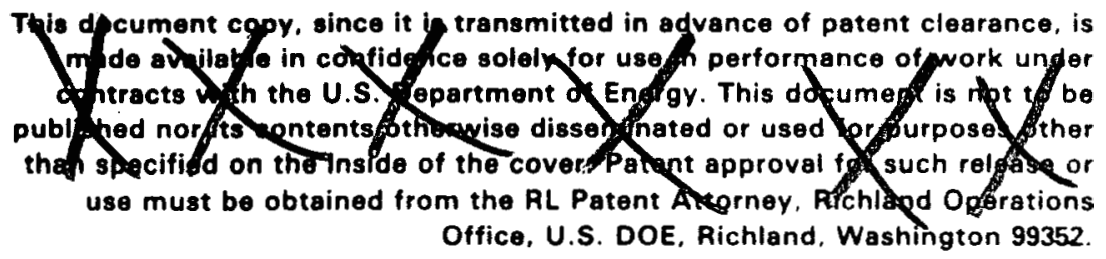

HANFORD ENGINEERING DEVELOPMENT LABORATORY

Operated by Westinghouse Hanford Company

P.0. Box 1970 Richland, WA 99352

A Subsidiary of Westinghouse Electric Corporation

Prepared for the U.S. Department of Energy under Contract No. EY-76-C-14-2170 


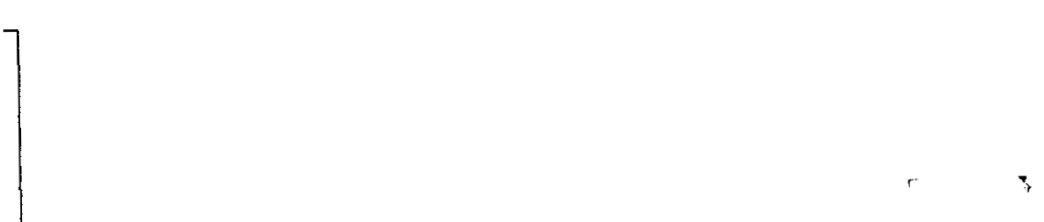

$\ldots$

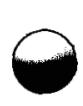

$\theta$ 


\title{
AS-BUILT DESCRIPTION OF THE EBR-II, RUN 97 DOSIMETRY EXPERIMENT
}

\author{
C. L. Long
}

J. A. U1 seth

E. P. Lippincott

\begin{abstract}
A dosimetry experiment has been designed and fabmcated for inclusion in the Experimental Breeder Reactor-II (EBR-II) during Run 97 in a Row 2 position. Various types of dosimeter material have been included in the single $B-7 c$ pin from $60 \mathrm{~cm}$ below midplane to $60 \mathrm{~cm}$ above. This report contains the as-built description of irradiation hardware and a detailed description of the dosimetry.
\end{abstract}




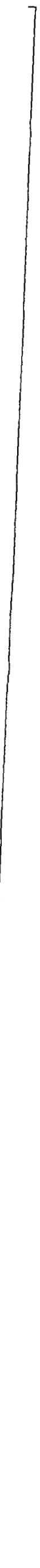

$\ominus$

$\bullet$ 


\section{CONTENTS}

\section{Page}

FIGURES

$v i$

TABLES

$v i$

I. INTRODUCTION

II. OBJECTIVES AND SCOPE 3

III. EXPERIMENT DESCRIPTION

A. GENERAL 5

B. PASSIVE DOSIMETERS 5

C. HELIUM ACCUMULATION FLUENCE MONITORS 12

D. SOLID STATE TRACK RECORDER SUBCAPSULES 13

$\begin{array}{ll}\text { E. EUROPEAN DOSIMETERS } & 13\end{array}$

$\begin{array}{ll}\text { BIBLIOGRAPHY } & 14\end{array}$

APPENDICES:

A - Run 97 Passive Dosimetry - Specific Details

B - Run 97 Helium Accumulation Fluence Monitors Specific Details

C - Run 97 Solid State Track Recorders - Specific Details

D - Run 97 European Dosimeters - Specific Details 


\section{FIGURES}

$\underline{\text { Page }}$

1 Run 97 Dosimeter Subcapsule Locations in the B-7c Pin Relative to Reactor Midplane.

2 Run 97 Spectral Set Subcapsules With Hole Numbering System. 7

3 Hollow Wire-Wrap Mockup Spectral Set Container and 9 Loading Order.

4 Gradient Set Subcapsule. $\quad 10$

5 Gradient Set Container Dimensions and Identification Scheme. 11

\section{TABLES}

1 Passive Monitor Material Description (Nominal) 8

2 Atomics International Container Loading 12 


\section{INTRODUCTION}

This report contains the as-built description of the Hanford Engineering Development Laboratory (HEDL) Dosimetry Experiment in Experimental Breeder Reactor No. II (EBR-II) to be irradiated during Run 97.* This experiment will produce information that will be useful for analysis of fuels and materials irradiations in EBR-II. The measurements are similar to those made during previous dosimetry tests irradiated during Runs $37 \mathrm{~F}^{1}, 50 \mathrm{H}^{2}$, and $75 \mathrm{D}^{3}$ at EBR-II. Unlike these earlier tests, the present test is more limited in scope and involves only one B-7c pin. The dosimeter encapsulation techniques and container designs used for the previous tests proved successful; therefore, they provide the basis for component design for this experiment.

*This experiment is being conducted in accordance with DOE-RRT Form 189, FF028, HEDL Task Authorization WGTVBA. 


\section{INTRODUCTION}

This report contains the as-built description of the Hanford Engineering Development Laboratory (HEDL) Dosimetry Experiment in Experimental Breeder Reactor No. II (EBR-II) to be irradiated during Run 97.* This experiment will produce information that will be useful for analysis of fuels and materials irradiations in EBR-II. The measurements are similar to those made during previous dosimetry tests irradiated during Runs $31 \mathrm{~F}^{1}, 50 \mathrm{H}^{2}$, and $75 \mathrm{D}^{3}$ at EBR-II. Unlike these earlier tests, the present test is more limited in scope and involves only one B-7c pin. The dosimeter encapsulation techniques and container designs used for the previous tests proved successfui; therefore, they provide the basis for component design for this experiment.

*This experiment is being conducted in accordance with DOE-RRT Form 189, FF028, HEDL Task Authorization WGTVBA. 
$\theta$

• 


\section{OBJECTIVES AND SCOPE}

In this experiment, a single dosimetry pin will be irradiated in EBR-II, Row 2, for one cycle. The dosimetry pin will contain a variety of dosimeters to meet the following objectives:

- Neutron Fluence Calibration. Measurement of the EBR-II Row 2 flux-spectrum will provide a calibration position for comparison with measurements from previous dosimetry tests to correlate variations due to changes in EBR-II loading. These data will be used, together with EBR-II calculations, to provide better fluence estimates for other EBR-II experiments, including fluence estimates for fuel cladding.

- Dosimetry Testing. Dosimetry types to be applied in the Fast Flux Test Facility (FFTF) will be tested in the high-power irradiation environment, including helium accumulation fluence monitors (HAFM) and niobium foil material of European origin.

In addition, fast-neutron spectrum background measurements will be made on solid state track recorder (SSTR) material proposed to be used for measurements in FFTF. 
$\theta$

。 


\section{EXPERIMENT DESCRIPTION}

A. GENERAL

The experiment requires that one Mark B-7C capsule, designated B-283, be irradiated in any Row 2 position for one reactor cycle. The dosimetry experiment is made up of 30 subcapsules containing different sets of dosimeters that are positioned from approximately $24 \mathrm{in} .(61 \mathrm{~cm})$ below to $24 \mathrm{in}$. $(61 \mathrm{~cm})$ above core midplane (see Figure 1). Stainless steel rod is used to position the subcapsules.

The 30 subcapsules consist of 8 fluence/spectral set subcapsules containing passive dosimeters, 16 gradient subcapsules containing $\mathrm{Fe}$ and $\mathrm{Co}$ passive dosimeters, 3 subcapsules containing HAFMs, and 3 subcapsules containing both SSTR sensors and niobium foil of European origin.

\section{B. PASSIVE DOSIMETERS}

Seven of the spectral set subcapsules are 0.746 in. $(1.89 \mathrm{~cm})$ in diameter and $0.50 \mathrm{in.}(1.27 \mathrm{~cm})$ long (see Figure 2). These cylinders have up to 19 holes of various diameters drilled axially into them. Stainless steel lids $0.060 \mathrm{in} .(0.154 \mathrm{~cm})$ thick are held in place by central screws. In this type of subcapsule, dosimeters will be irradiated at the same level for exact comparisons. See Table 1 for a general description of the passive monitor materials. 

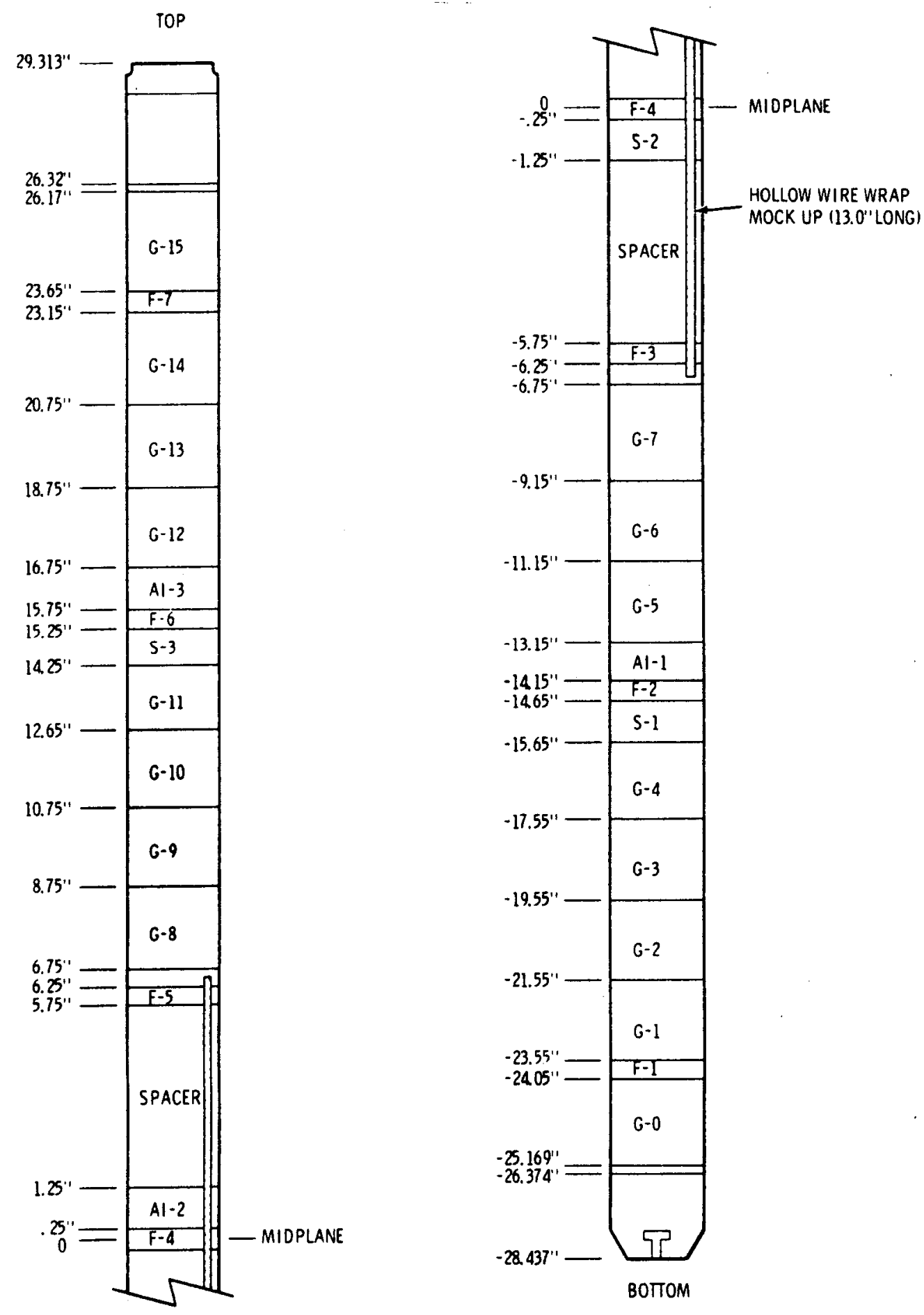

NOIL 1. THESE DIMENSIONS WERE OBTAINED FROM HEDL H-3-4407I SHEET I AND SHEET 2

NOTE 2. G DENOTES GRADIENT SET, F DENOTES FLUENCE SET, AI DENOTES ATOMIC INTERNATIONAL DOSIMETER PACKAGES AND S DENOTES SSTR PACKAGES

FIGURE 1. Run 97 Dosimeter Subcapsule Locations in the B-7C Pin Relative to Reactor Midplane. 

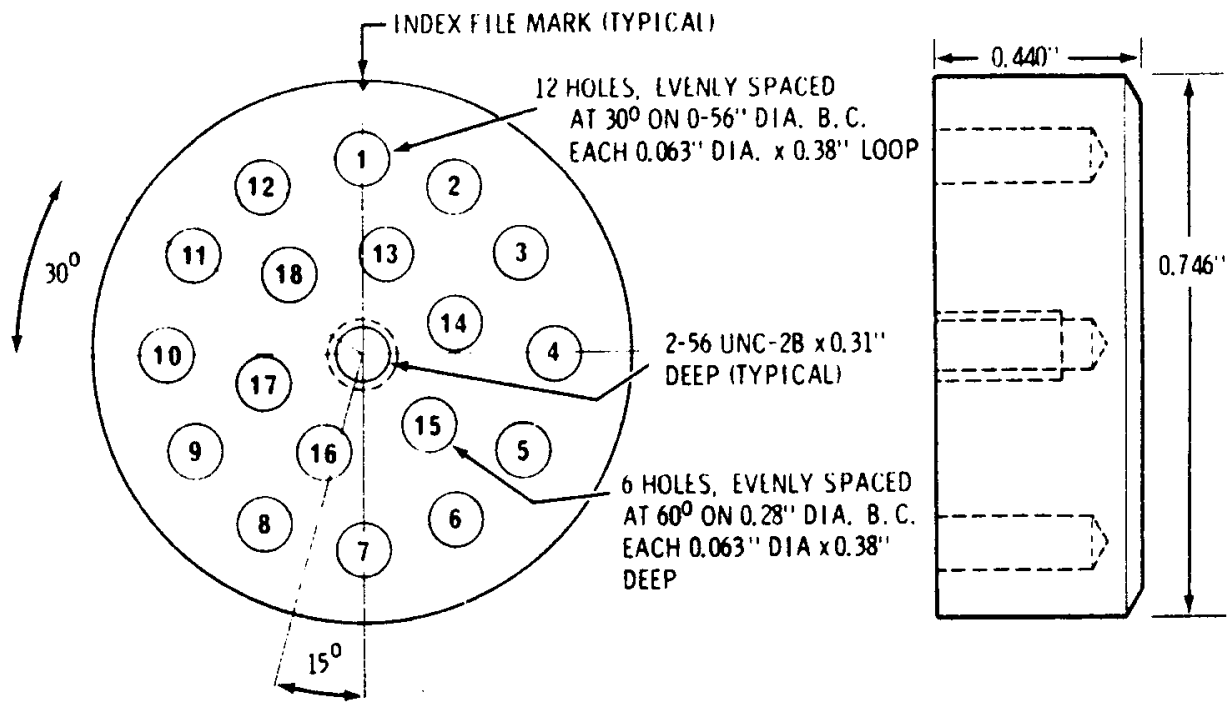

SPECTRAL

SET

10

$F-1$

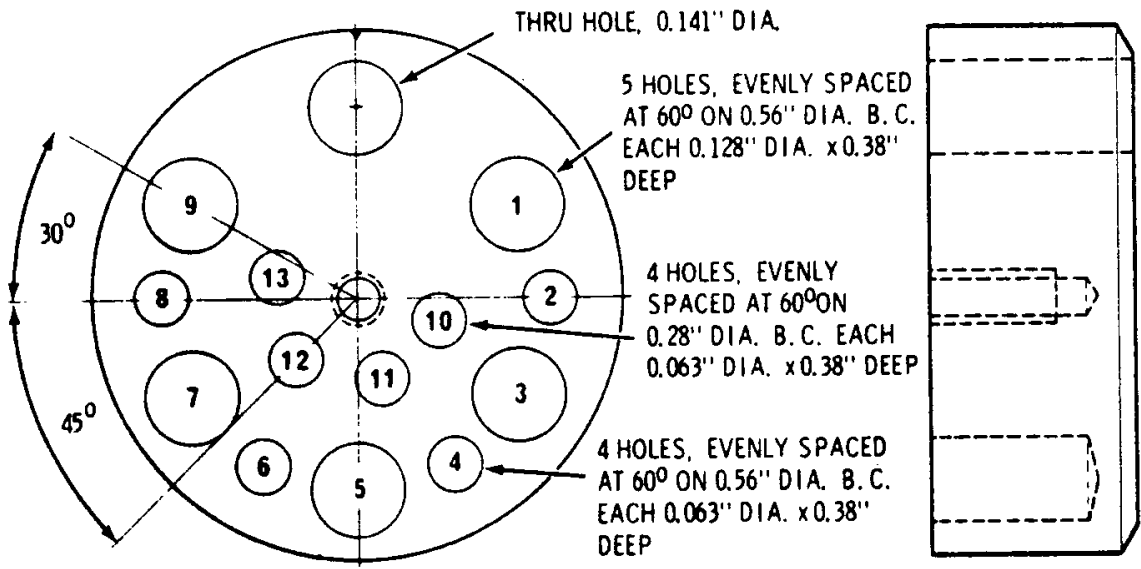

$F-3$

$F-4$

$F-5$
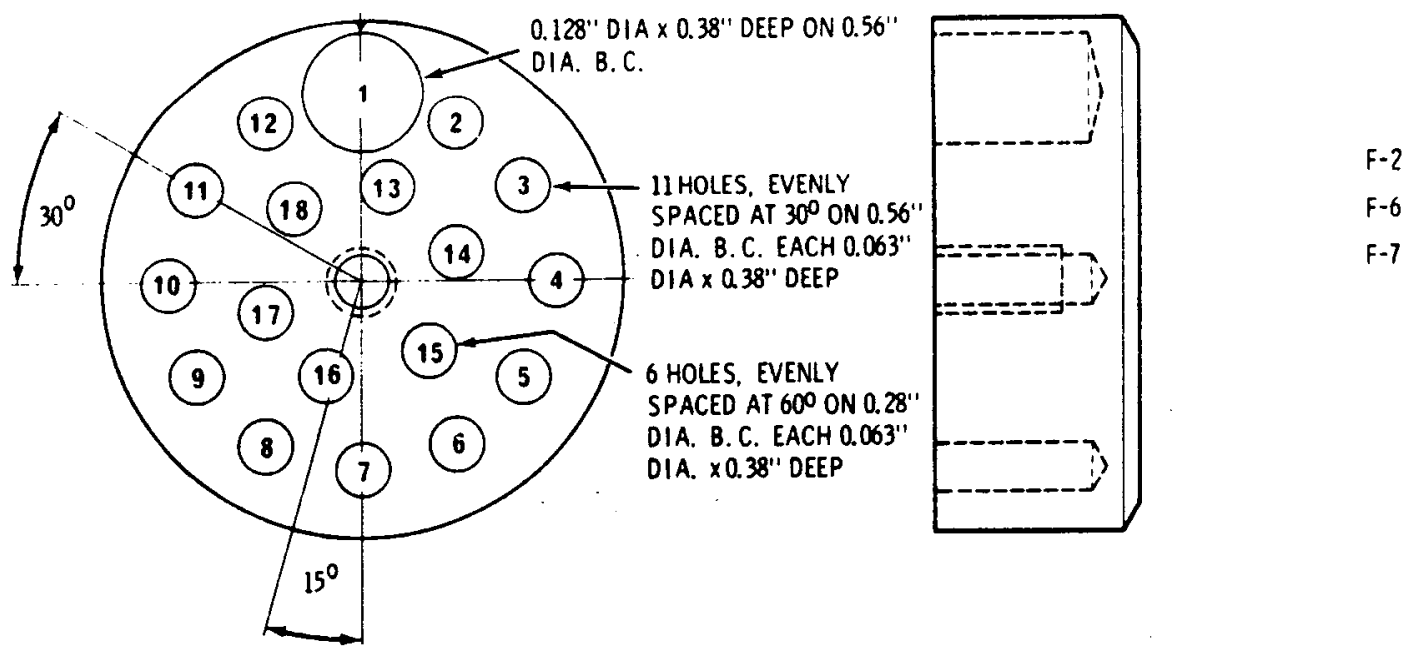

FIGURE 2. Run 97 Spectral Set Subcapsules With Hole Numbering System. 
TABLE 1

PASSIVE MONITOR MATERIAL DESCRIPTION (NOMINAL)

\begin{tabular}{|c|c|c|c|c|c|}
\hline & Form & $\begin{array}{r}\text { Capsule } \\
\frac{\text { (i }}{0.050-\text { in. OD }}\end{array}$ & $\begin{array}{l}\text { Length } \\
0.035-i n .00\end{array}$ & $\begin{array}{l}\text { Bare Length } \\
\text { (in.) }\end{array}$ & $\begin{array}{c}\text { Material Mass } \\
(\mathrm{mg})\end{array}$ \\
\hline $235 \mathrm{UO}_{2}$ & Oxide Wire & 0.125 & 0.190 & -- & 1.6 \\
\hline $23{ }^{8} \mathrm{UO}_{2}$ & Oxide Wire & 0.315 & 0.310 & -- & 6.0 \\
\hline $2{ }^{2} \mathrm{NpO}_{2}$ & Oxide Wire & 0.345 & 0.280 & -- & 4.5 \\
\hline $23{ }^{9} \mathrm{PuO}_{2}$ & Oxide Wire & 0.285 & 0.250 & -- & 1.4 \\
\hline $10 \mathrm{~B}$ & Natural Boron Crystals & 0.255 & 0.250 & -- & 0.08 \\
\hline${ }^{6} \mathrm{LiF}$ & Natural Lif Crystals & 0.255 & 0.250 & -- & 0.10 \\
\hline $\mathrm{Sc}_{2} \mathrm{O}_{3}$ & Ceramic Wire & 0.255 & 0.340 & -- & 1.3 \\
\hline $1.0 \% \operatorname{Cov}$ & Metal Wire & - & 0.270 & 0.10 & 2.0 \\
\hline $0.1 \%$ CoMgo & Ceramic Wire & - & 0.300 & -- & 1.3 \\
\hline $0.1 \% \operatorname{CoAl}$ & Metal Wire & - & 0.240 & 0.10 & 1.3 \\
\hline $0.1 \%$ AgA 1 & Metal Wire & - & 0.210 & - & 1.3 \\
\hline $0.1 \%$ AuA 1 & Metal Wire & - & 0.180 & -- & 1.2 \\
\hline $\mathrm{MnO}_{2}$ & Ceramic Wire & - & 0.240 & -- & 1.5 \\
\hline $0.1 \% \mathrm{TaAl}$ & Metal Wire & - & 0.240 & 0.10 & 0.7 \\
\hline $0.033-i n$. diam SS & Metal Wire & - & - & 0.10 & 10.0 \\
\hline Nb & Foil and Wire & - & 0.240 & 0.12 & 4.5 \\
\hline $\mathrm{Fe}$ & Metal Wire & - & 0.240 & 0.10 & 4.0 \\
\hline Ni & Metal Wire & - & 0.180 & 0.10 & 2.8 \\
\hline $\mathrm{Cu}$ & Metal Wire & - & 0.210 & 0.10 & 4.0 \\
\hline$T i$ & Metal Wire & - & 0.240 & 0.10 & 2.1 \\
\hline V Blank & WeIded Tube & 0.255 & - & -. & 35.0 \\
\hline $0.1 \% \mathrm{Sc}_{2} \mathrm{O}_{3}$ & Ceramic Wire & - & 0.300 & - & 1.0 \\
\hline $1.0 \% \mathrm{TaV}$ & Metal Wire & - & - & 0.10 & 3.0 \\
\hline
\end{tabular}

The eighth spectral set subcapsule consists of a hollow wire-wrap tube [0.056-in. $(0.142-\mathrm{cm})$ OD by $0.046-$ in.$(0.117-\mathrm{cm})$ ID by $13.0-$ in. $(33.0-\mathrm{cm})$ length] that contains a spectral set composed of $0.035-\mathrm{in} .(0.089-\mathrm{cm})$ vanadium encapsulated monitors (see Figure 3 ). The dosimeters will be centered at midplane and will be used for direct comparison with a regular spectral set located at midplane. This type of dosimetry has been attached to FFTF fuel pins for Cycle 1 irradiation. The wire-wrap tube passes through 0.140-in. $(0.356-\mathrm{cm})$ diameter holes drilled through all dosimeter, HAFM, and SSTR subcapsules in the core region (see Figure 1). Tables $A-1$ through $A-8$ in Appendix A specifically describe the monitors used in each spectral set. 


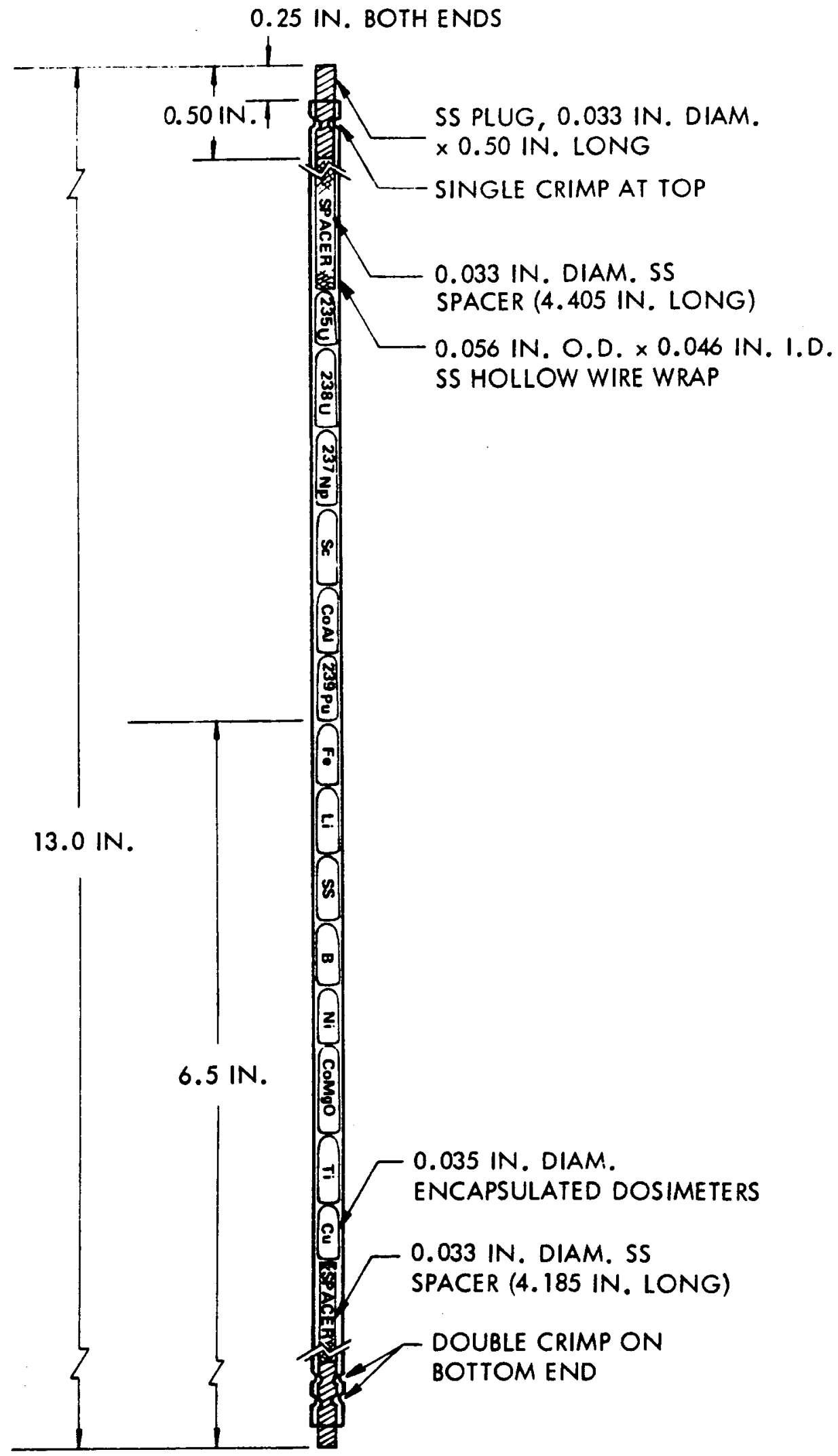

FIGURE 3. Hollow Wire-Wrap Mockup Spectral Set Container and Loading Order. 
These tables list the traceability of each material by purchase order and batch number, physical dimensions, material weight, and identification information.

The 16 gradient sets used in this experiment are contained in stainless steel rods that have a central hole drilled into them (see Figure 4). Since these rods also are used as spacers to properly position the other subcapsules, they are of varying lengths as shown. The gradient set subcapsules are shown in Figure 5, which gives the dimension, loading order, and capsule identification method. Table A-9 describes the monitors in each gradient set in greater detail.

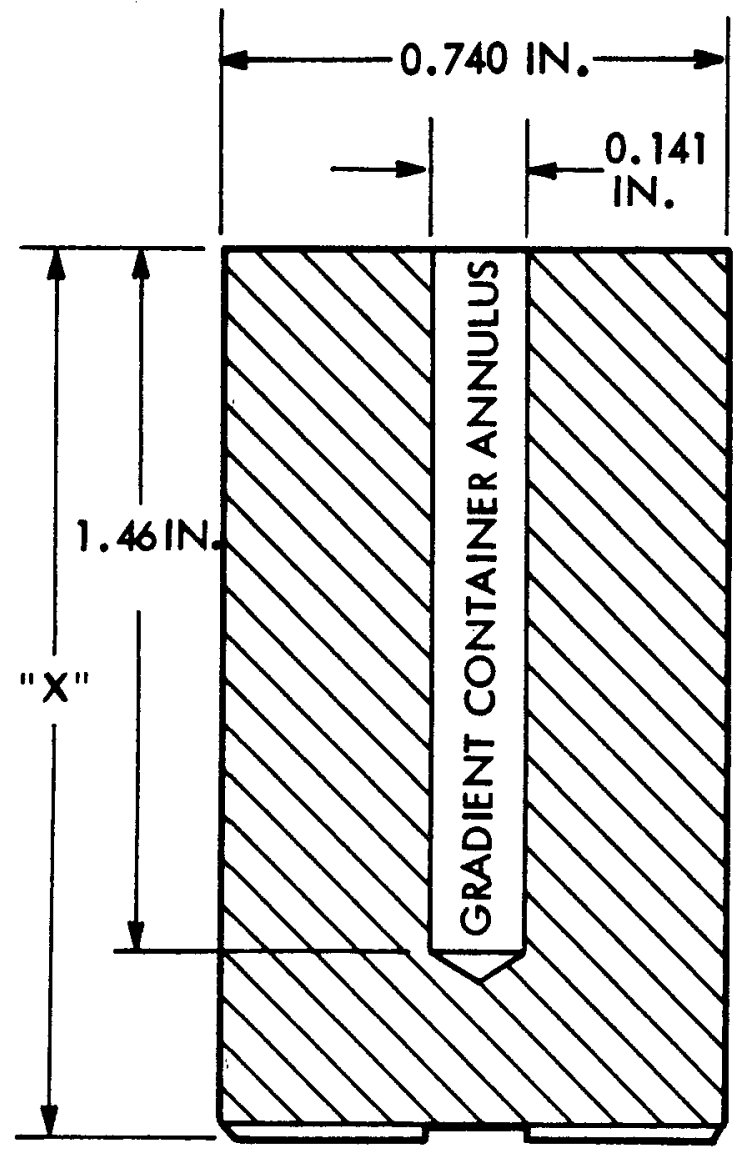

\begin{tabular}{|c|c|}
\hline $\begin{array}{c}\text { GRADIENT } \\
\text { SET ID }\end{array}$ & $\begin{array}{c}\text { DIMENSION } \\
\text { "X" }\end{array}$ \\
\hline G-11 & 1.600 \\
\hline G-4, G-10 & 1.900 \\
\hline G-1, G-2 \\
G-3, G-5 \\
G-6, G-8 \\
G-9, G-12 \\
G-13 & 2.000 \\
\hline G-0 & 2.119 \\
\hline G-7, G-14 & 2.400 \\
\hline G-15 & 2.520 \\
\hline
\end{tabular}

FIGURE 4. Gradient Set SubcapsuTe. 

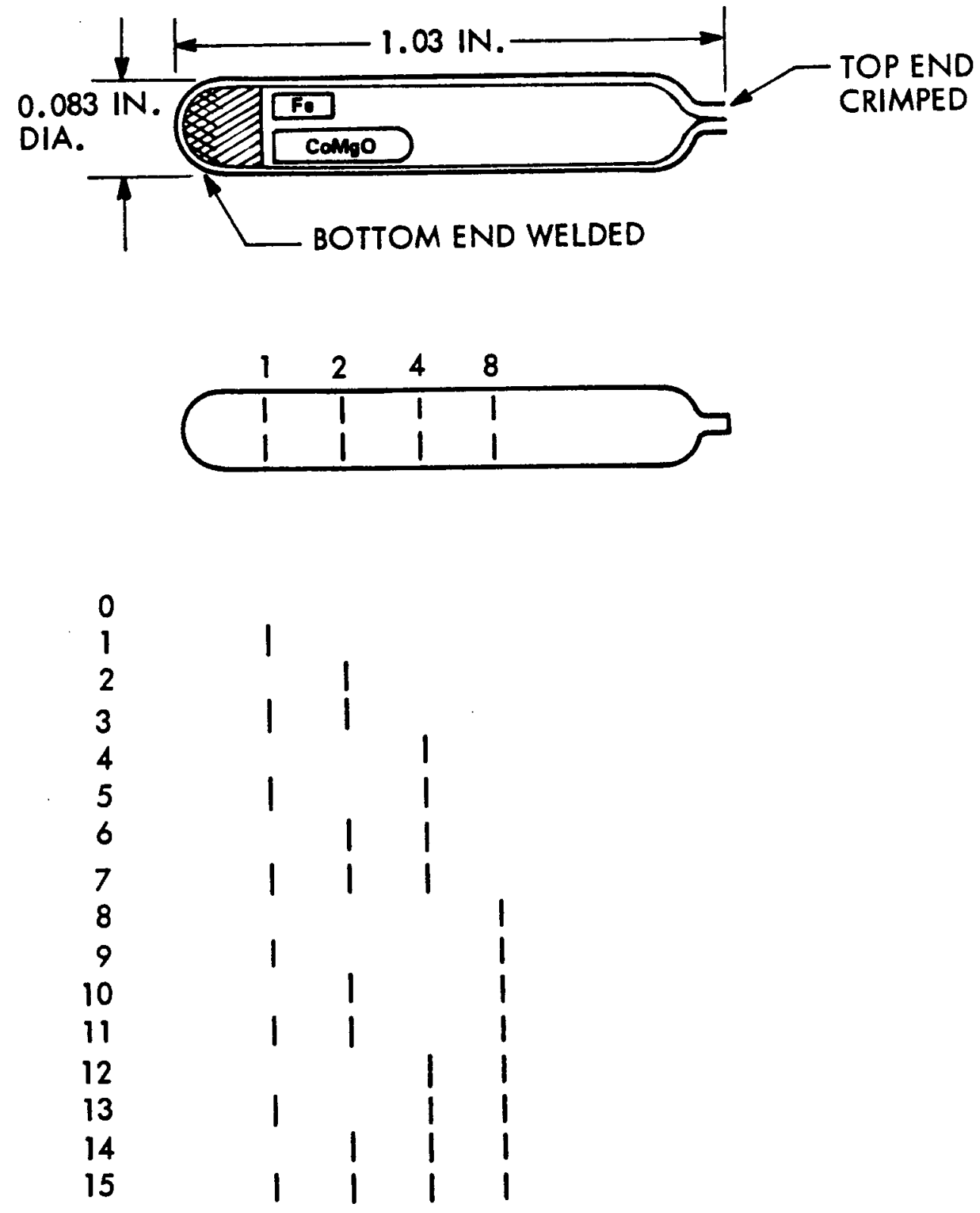

FIGURE 5. Gradient Set Container Dimensions and Identification Scheme. 
C. HELIUM ACCUMULATION FLUENCE MONITORS

Specific information for all HAFMs is given in Appendix B. The HAFM subcapsules are right circular cylinders. When capped with an 0.060-in. $(0.152-\mathrm{cm})$ thick stainless steel 1 id, they are $1.00 \mathrm{in.}(2.54 \mathrm{~cm})$ long by $0.746-$ in. $(1.89-\mathrm{cm})$ diameter, similar to the spectral set dosimetry subcapsules (see Figure B-1). Three holes are drilled axially into the cylinders. Two of the holes are $0.141 \mathrm{in} .(0.358 \mathrm{~cm})$ in diameter and contain unsealed containers holding bare wires of vanadium, niobium, platinum, and stainless steel; the larger hole, $0.453-$ in. $(1.15-\mathrm{cm})$ diameter, contains sealed containers holding material similar to that in the smaller holes. Table 2 lists the positioning and identification of the nine capsules furnished by Atomics International (AI).

TABLE 2

ATOMICS INTERNATIONAL CONTAINER LOADING

Container

Identification

$A I-1$

$A I-2$

AI -3
Container

Hole Number

1

2

3

1

3

4

1

3

4
AI Capsule (a)

E

N

C

H

K

A

0

$P$

B

$\overline{(a)}$ See Appendix B for contents of AI capsules. 


\section{SOLID STATE TRACK RECORDER SUBCAPSULES}

Detailed information on the SSTR materials is listed in Appendix C. Each of the SSTR subcapsules (also shown in Figure B-1) contains six natural quartz crystal SSTRs and two pieces of backing material made of high purity nickel. The loading arrangement (1 isted in Tables $\mathrm{C}-1$ and $\mathrm{C}-2$ ) gives two quartz-nickel interfaces to monitor the fissionable content of the nickel and several types of quartz-quartz interfaces. The containers are sealed (welded) and placed into the large hole of each subcapsule.

\section{E. EUROPEAN DOSIMETERS}

Detailed information on the European niobium dosimeters is listed in Appendix D. Niobium foils of Belgian, French, and German origin [0.0008 in. $(0.002 \mathrm{~cm})$ thick by $0.118-\mathrm{in} .(0.289-\mathrm{cm})$ diameter] were interleaved with titanium spacers $[0.001$ in. $(0.0025 \mathrm{~cm})$ thick by $0.125-\mathrm{in} .(0.3175-\mathrm{cm})$ diameter], loaded into a stainless steel container [0.150-in. $(0.381-\mathrm{cm})$ OD by $0.130-$ in. $(0.330-\mathrm{cm})[D]$, and placed on top of the SSTR containers in two of the SSTR subcapsules, $S-1$ and S-2 (see Tables D-1 and D-2). 


\section{BIBLIOGRAPHY}

Lippincott, E. P., et al. "EBR-II 75D High-Power Dosimetry Test - Preliminary Report," HEDL-TME 75- $\frac{6}{86}$, Hanford Engineering Development Laboratory, 1975.

McElroy, W. N., et al. "EBR-II Dosimetry Test Data Analysis (Reactor Runs $31 E$ and 3IF)," BNWL-1402, Battelle, Northwest Laboratory, 1970.

McElroy, W. N., et al. "EBR-II Dos imetry Test (Reactor Runs 50G and 50H)," HEDL-TME 73-62, Hanford Engineering Development Laboratory, 1973. 


\section{APPENDIX A}

RUN 97 PASSIVE DOSIMETRY - SPECIFIC DETAILS 
TABLE A-1

RUN 97, CONTAINER F-1, SPECTRAL SET DETAILS

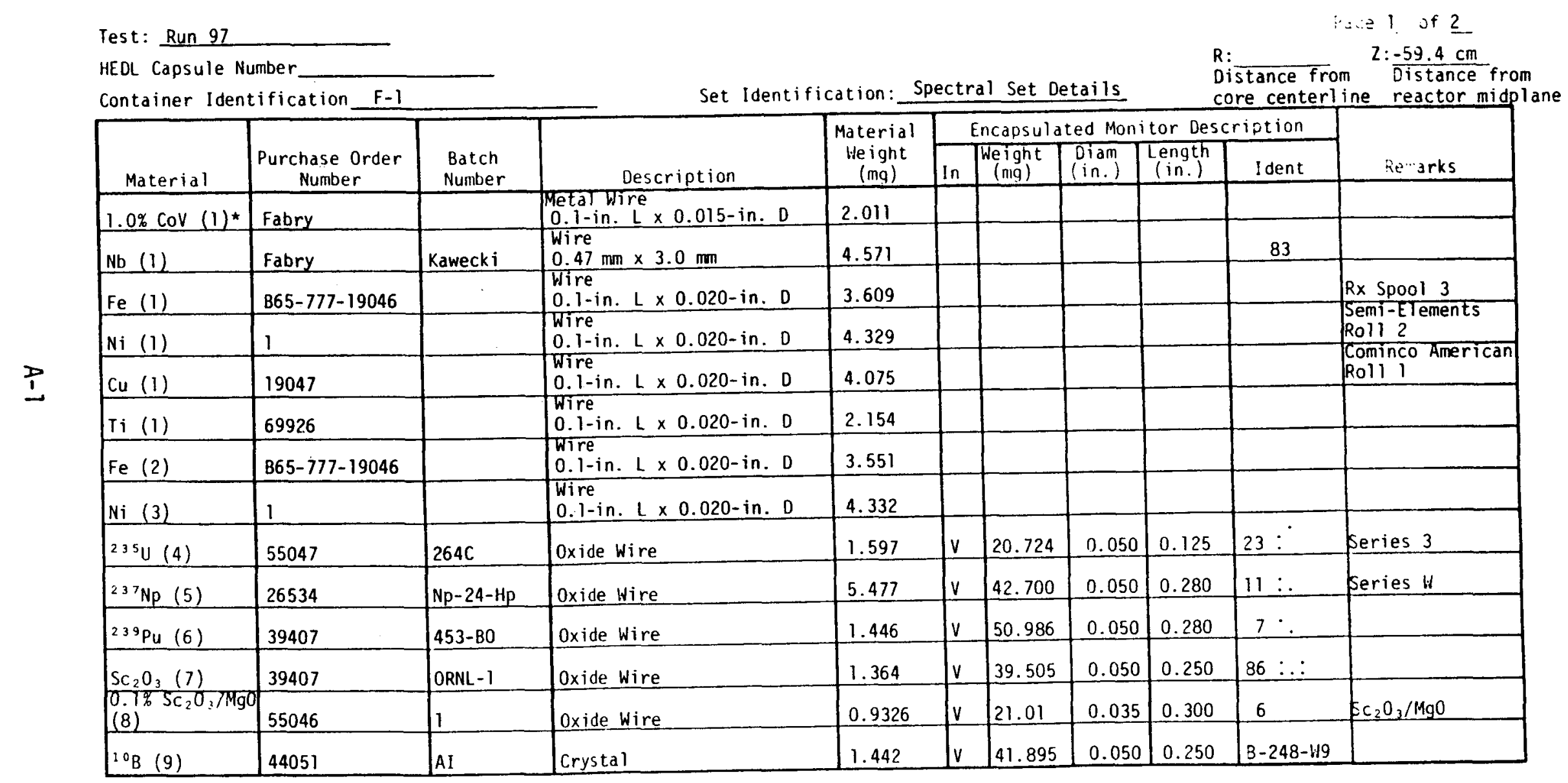

* Number in parentheses $=$ hole number in container.

Container and lid SS heat $\$ 68876$. 
TABLE A-1 (Continued)

Test: $\quad$ Run 97

HEDL Capsule Number

Container Identification $\mathrm{F}-1$

Set Identification: Spectral Set Details

Page 2 of 2

\begin{tabular}{|c|c|c|c|c|c|c|c|c|c|c|}
\hline \multirow[b]{2}{*}{ Material } & \multirow[b]{2}{*}{$\begin{array}{c}\text { Purchase Order } \\
\text { Number }\end{array}$} & \multirow[b]{2}{*}{$\begin{array}{l}\text { Batch } \\
\text { Number }\end{array}$} & \multirow[b]{2}{*}{ Description } & \multirow{2}{*}{$\begin{array}{c}\text { Material } \\
\text { Weight } \\
\text { (mg) }\end{array}$} & \multicolumn{5}{|c|}{ Encapsulated Monitor Description } & \multirow[b]{2}{*}{ Remarks } \\
\hline & & & & & In & $\begin{array}{c}\text { Weight } \\
\text { (mg) }\end{array}$ & $\begin{array}{l}\text { Diam } \\
\text { (in.) } \\
\end{array}$ & $\begin{array}{l}\text { Length } \\
\text { (in.) }\end{array}$ & Ident & \\
\hline${ }^{6} \mathrm{LiF}(10)$ & 40051 & AI & Crystal & 2.367 & lv & 40.433 & 0.050 & 0.255 & L203-Fe & \\
\hline $1.0 \% \operatorname{Cov}(11)$ & Fabry & & $\begin{array}{l}\text { Metal Wire } \\
0.1-\text { in. } L \times 0.015-i n . D\end{array}$ & 2.083 & & & & & & \\
\hline (ii) & 27940 & ORNL Tube 1 & $\begin{array}{l}\text { Oxide Wire } \\
0.100-\text { in. } L \times 0.020-i n . D\end{array}$ & 1.528 & $\mathrm{v}$ & 24.84 & 0.035 & 0.300 & $x$ & Series 2 \\
\hline $0.1 \% \operatorname{COAl}(11)$ & & SPM-953 & $\begin{array}{l}\text { Metal Wire } \\
0.100-\text { in. } L \times 0.020 \text {-in. } D\end{array}$ & 1.2856 & & & & & & Roll 4 NBS \\
\hline $1.0 \% \operatorname{Cov}(12)$ & Fabry & & $\begin{array}{l}1.0 \% \text { CoV Wire } \\
0.1-\text { in. } L \times 0.015-i n .0\end{array}$ & 1.8986 & & & & & & \\
\hline $\mathrm{Ti}(12)$ & 69926 & & $\begin{array}{l}\text { Wire } \\
0.1 \text {-in. } L \times 0.020 \text {-in. } 0\end{array}$ & 2.219 & & & & & & \\
\hline $0.1 \% \mathrm{AgAl}$ (13) & $03783^{\circ}$ & Rx Wire & Metal Wire & 1.2675 & $v$ & 17.459 & 0.035 & 0.210 & 4 & \\
\hline $0.1 \%$ AUAl (14) & & Rx Wire & Metal Wire & 1.2340 & lv & 13.788 & 0.035 & 0.210 & $F$ & \\
\hline $\mathrm{Mn}_{3} \mathrm{O}_{4}$ (1.5) & 55086 & & $\begin{array}{l}\text { 0xide Wire } \\
0.125-i n . L \times 0.020-i n . D\end{array}$ & 1.4535 & v & 18.818 & 0.035 & 0.240 & $\mathrm{~J}$ & \\
\hline $0.1 \%$ TaAl (16) & 03783 & $217 \mathrm{~W}$ & $\begin{array}{l}\text { Metal Wire } \\
0.100-i n . L \times 0.020-i n . D\end{array}$ & 0.6775 & & & & & & \\
\hline $1.0 \% \operatorname{TaV}(16)$ & 32548 & ORNL & $0.100-$ in. $L \times 0.020$-in. $D$ & 2.969 & & & & & & \\
\hline SS Wire (17) & & & $0.1-$ in. $L \times 0.033-$ in. $D$ & 9.890 & & & & & & FOTA SS Rod \\
\hline Cu (18) & 19047 & & $\begin{array}{l}\text { Wire } \\
0.100-\text { in. } L \times 0.020 \text {-in. } D\end{array}$ & 4.047 & & & & & & \\
\hline
\end{tabular}


TABLE A-2

RUN 97, CONTAINER F-2, SPECTRAL SET DETAILS

Test: Run 97

HEDL Capsule Number

Container Identification

$\mathrm{F}-2$

Set Identification:

Page 1 of 4

\begin{tabular}{|c|c|c|c|c|c|c|c|c|c|c|}
\hline \multirow[b]{2}{*}{ Material } & \multirow[b]{2}{*}{$\begin{array}{c}\text { Purchase Order } \\
\text { Number }\end{array}$} & \multirow[b]{2}{*}{$\begin{array}{l}\text { Batch } \\
\text { Number }\end{array}$} & \multirow[b]{2}{*}{ Description } & \multirow{2}{*}{$\begin{array}{l}\text { Material } \\
\text { He ight } \\
\text { (mg) }\end{array}$} & \multicolumn{5}{|c|}{ Encapsulated Monitor Description } & \multirow[b]{2}{*}{ Remarks } \\
\hline & & & & & In & $\begin{array}{c}\text { Weight } \\
(\text { mig) }\end{array}$ & $\begin{array}{l}\text { Diam } \\
(\text { in. }) \\
\end{array}$ & $\begin{array}{l}\text { Length } \\
\text { (in.) }\end{array}$ & Ident & \\
\hline $235 U(1)^{*}$ & 55047 & $264-C$ & Oxide Wire & 1.389 & $v$ & 21.060 & 0.050 & 0.125 & $1 \cdot$ & Series 3 \\
\hline${ }^{238} \mathrm{U}(1)$ & 39407 & $E S-Z$ & Oxide Wire & 7.233 & $v$ & 51.352 & 0.050 & 0.310 & $37 \vdots$ & Series I \\
\hline $23^{3} \mathrm{~Np}(1)$ & 39407 & $\mathrm{~Np}-24-\mathrm{Hp}$ & Metal Wire & 5.878 & $v$ & 51.474 & 0.050 & 0.345 & $21 \div$ & Series 1 \\
\hline $1.0 \% \operatorname{CoV}(1)$ & Fabry & . & $0.1-$ in. $L \times 0.015$-in. $D$ & 2.095 & & & & & & \\
\hline $\mathrm{Nb}(1)$ & Fabry & Kawecki & $0.47 \mathrm{~mm} \times 3.0 \mathrm{~mm}$ & 4.268 & & & & & 79 & \\
\hline Fe (1) & B65-777- 19046 & & $\begin{array}{l}\text { Metal wire } \\
0.1-\text { in. } L \times 0.020-\text { in. } D\end{array}$ & 3.947 & & & & & & \\
\hline $\mathrm{Fe} \mathrm{(1)}$ & $865-777-19046$ & & $\begin{array}{l}\text { Metal Wire } \\
0.1 \text {-in. } L \times 0.020-\text { in. } D\end{array}$ & 4.027 & & & & & & \\
\hline $\mathrm{Ni}(1)$ & 1 & & $\begin{array}{l}\text { Meta Wire } \\
0.1 \text {-in. L } \times 0.020 \text {-in. D }\end{array}$ & 4.389 & & & & & & \\
\hline $\mathrm{Cu}(1)$ & 19047 & & $\begin{array}{l}\text { Metal Wire } \\
0.1 \text {-in. L } \times 0.020-i n .0\end{array}$ & 4.163 & & & & & & \\
\hline Ti (1) & 69926 & & $\begin{array}{l}\text { Metal Wire } \\
0.1 \text {-in. L } \times 0.020-\text { in. D }\end{array}$ & 2.146 & & & & & & \\
\hline $235 \mathrm{U}(2)$ & 55047 & $264-C$ & Oxide Wire & 1.583 & v & 20.874 & 0.050 & 0.125 & $51 \ldots$ & Series 3 \\
\hline $1.0 \% \operatorname{cov}(2)$ & Fabry & & $\begin{array}{l}\text { Metal Wire } \\
0.1-\text { in. } L \times 0.015-i n . D\end{array}$ & 1.9117 & & & & & & \\
\hline $\mathrm{Fe}(2)$ & B65-777-19046 & & $\begin{array}{l}\text { Meta Wire } \\
0.1-i n . L \times 0.020-i n . D\end{array}$ & 3.719 & & & & & & \\
\hline $\mathrm{Fe}(3)$ & 19046 & $S C-53$ & $\begin{array}{l}\text { Metal Wire } \\
0.1 \text {-in. L } ~\end{array}$ & 4.047 & $v$ & 20.55 & 0.035 & 0.240 & $T$ & Series 5 \\
\hline
\end{tabular}

*Number in parentheses $=$ hole number in container. 


\section{TABLE A-2 (Continued)}

Test: Run 97

HEDL Capsule Number

Container Identificatic.

$\mathrm{F}-2$

Set Identification:

Page 2 of 4

\begin{tabular}{|c|c|c|c|c|c|c|c|c|c|c|}
\hline \multirow[b]{2}{*}{ Material } & \multirow[b]{2}{*}{$\begin{array}{c}\text { Purchase }=r \text { rer } \\
\text { Numb }=r\end{array}$} & \multirow[b]{2}{*}{$\begin{array}{l}\text { Batch } \\
\text { Number }\end{array}$} & \multirow[b]{2}{*}{ Description } & \multirow{2}{*}{$\begin{array}{c}\text { Material } \\
\text { Weight } \\
\text { (mg) }\end{array}$} & \multicolumn{5}{|c|}{ Encapsulated Monitor Description } & \multirow[b]{2}{*}{ Remlarks } \\
\hline & & & & & In & $\begin{array}{c}\text { Weight } \\
\text { (miq) }\end{array}$ & $\begin{array}{l}\text { Diam } \\
\text { (in.) }\end{array}$ & $\begin{array}{c}\text { Length } \\
\text { (in.) }\end{array}$ & Ident & \\
\hline $\mathrm{Fe}(3)$ & 19046 & & $\begin{array}{l}\text { Thetal Wire } \\
0 . \text {-in. } L \times 0.020 \text {-in. } 0\end{array}$ & 3.997 & & & & & & \\
\hline $\mathrm{SC}_{2} \mathrm{O}_{3}$ (4) & 39407 & ORNL-1 & Oxide Wire & 1.428 & v & |42.291 & 0.050 & 0.255 & $91 \ldots:$ & \\
\hline $\mathrm{Fe}(4)$ & 19046 & & $\begin{array}{l}\text { Metal Wire } \\
0.1-i n . L \times 0.020-i n .0\end{array}$ & 3.754 & & & & & & \\
\hline $\begin{array}{l}0 .{ }^{10} \mathrm{Sc}_{2} \mathrm{O}_{3} / \mathrm{MgC} \\
(5)\end{array}$ & 55046 & 1 & $\begin{array}{l}\text { Oxide wire } \\
0.125-\text { in. } L \times 0.020-\text { in. } D\end{array}$ & 0.9010 & v & 21.91 & 0.035 & 0.300 & 1 & \\
\hline $\mathrm{Fe}(5)$ & 19046 & & $\begin{array}{l}\text { Metal wire } \\
0.100-\text { in. } L \times 0.020-i n . D\end{array}$ & 3.694 & & & & & & \\
\hline $1{ }^{1} \mathrm{~B}(6)$ & 44051 & AI & Crystal & 1.301 & $v$ & 41.298 & 0.050 & 0.255 & B-241-T9 & \\
\hline Fe (6) & 19046 & & $\begin{array}{l}\text { Metal Wire } \\
0.1-\text { in. } L \times 0.020-\text { in. } 0\end{array}$ & 3.753 & & & & & & \\
\hline${ }^{6} \mathrm{LiF}(7)$ & 44051 & AI & Crystal & 2.974 & v & 43.020 & 0.050 & 0.255 & L215-Al & \\
\hline $\mathrm{Fe}(7)$ & 19046 & & $\begin{array}{l}\text { Metal Wire } \\
0.1-i n . L \times 0.020-i n . D\end{array}$ & 3.983 & & & & & & \\
\hline $\mathrm{Fe} \mathrm{(8)}$ & 19046 & & $\begin{array}{l}\text { Metal Wire } \\
0.1-i n . L \times 0.020-i n . D\end{array}$ & 3.799 & & & & & & \\
\hline $\mathrm{Cu}(8)$ & 19047 & & $\begin{array}{l}\text { Metal Wire } \\
0.1-i n . L \times 0.020-i n . D\end{array}$ & 4.078 & & & & & & \\
\hline $\mathrm{Ti}(8)$ & 69926 & & $\begin{array}{l}\text { Metal Wire } \\
0.1-i n . L \times 0.020-i n .0\end{array}$ & 2.136 & & & & & & \\
\hline $\mathrm{Fe}(9)$ & 19046 & & $\begin{array}{l}\text { Metal Wire } \\
0.1-i n . L \times 0.020-i n . D\end{array}$ & 4.037 & & & & & & \\
\hline Ni (9) & 1 & & $\begin{array}{l}\text { Metal Wire } \\
0.1-i n . L \times 0.020-i n .0\end{array}$ & 4.371 & & & & & & \\
\hline
\end{tabular}




\section{TABLE A-2 (Continued)}

F-2

\begin{tabular}{|c|c|c|}
\hline Material & $\begin{array}{c}\text { Purchase Order } \\
\text { Number }\end{array}$ & $\begin{array}{l}\text { Batch } \\
\text { Number }\end{array}$ \\
\hline $1.0 \% \operatorname{Cov}(10)$ & Fabry & \\
\hline $\begin{array}{l}0.1 \% \text { CoMgO } \\
\text { (10) }\end{array}$ & 27940 & ORNL Tube \\
\hline $\begin{array}{l}0.1 \% \operatorname{CoA} 1 \\
(10)\end{array}$ & & SRM-953 \\
\hline $\mathrm{Fe}(10)$ & 19046 & \\
\hline \multicolumn{3}{|l|}{ Ss (11) } \\
\hline $\mathrm{Fe}(11)$ & 19046 & \\
\hline $2{ }^{38} \mathrm{U} \quad(12)$ & 39407 & ES-2 \\
\hline $23^{7} \mathrm{~Np}$ (13) & 39407 & $\mathrm{~Np}-24-\mathrm{Hp}$ \\
\hline \multicolumn{3}{|l|}{$0.1 \%$ AuAl (14) } \\
\hline $\mathrm{Fe} \mathrm{(14)}$ & 19046 & Rx Wire \\
\hline $0.1 \%$ AgAl (15) & 03783 & Rx Wire \\
\hline $\mathrm{Fe}(15)$ & 19046 & \\
\hline $\mathrm{MnO}_{2}$ (16) & 55086 & \\
\hline (16) & 19046 & \\
\hline
\end{tabular}

Test: Run 97

HEOL Capsule Number

Container Identification
Set Identification:

age 3 of 4

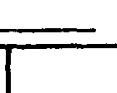

Description.

\section{Metal Wire} 0xide Wire

$L \times 0.020-$ in 0

Metal Wire

$0.100-i n . L \times 0.020-i n . D \quad 1.329$

Metal Wire

0.1 -in. L $\times 0.020$-in. D 3.788

$\begin{array}{lll}0.1-i n . L \times 0.033-i n . D & 9.103\end{array}$

Metal Wire

0.1 -in $L \times 0.020$-in. 0

\begin{tabular}{l}
0.742 \\
\hline
\end{tabular}

Oxide Wire

Oxide Wire

Metal Wire

Metal wire

0.1 -in. L $\times 0.020-$ in.

\begin{tabular}{|c|c|c|c|c|c|c|}
\hline \multirow{2}{*}{$\begin{array}{c}\text { Material } \\
\text { Weight } \\
\text { (mg) }\end{array}$} & \multicolumn{5}{|c|}{ Encapsulated Monitor Description } & \multirow[b]{2}{*}{ Remarks } \\
\hline & $\ln$ & $\begin{array}{c}\text { We ight } \\
\text { (mg) }\end{array}$ & $\begin{array}{l}\text { Diam } \\
\text { (in.) }\end{array}$ & $\begin{array}{c}\text { Tength } \\
\text { (in.) }\end{array}$ & Ident & \\
\hline \multicolumn{7}{|l|}{2.064} \\
\hline 1.171 & $v$ & 20.16 & 0.035 & 0.300 & $\mathrm{~F}$ & Series 4 \\
\hline \multicolumn{7}{|l|}{1.3291} \\
\hline \multicolumn{7}{|l|}{3.788} \\
\hline 9.103 & & & & & & FOTA SS-Wire \\
\hline \multicolumn{7}{|l|}{3.742} \\
\hline 7.403 & v & 50.803 & 0.050 & 0.315 & $13:$ & Series I \\
\hline 6.592 & $v$ & 51.109 & 0.050 & 0.345 & $22:$ & Series 1 \\
\hline 1.3525 & $v$ & 14.878 & 0.035 & 0.180 & $H$ & \\
\hline \multicolumn{7}{|l|}{3.987} \\
\hline 1.2065 & $v$ & 16.461 & 0.035 & 0.210 & 2 & \\
\hline \multicolumn{7}{|l|}{3.809} \\
\hline 1.3938 & v & 18.331 & 0.035 & 0.240 & $k$ & \\
\hline 3.799 & & & & & & \\
\hline
\end{tabular}


TABLE A-2 (Continued)

Test: Run 97

HEOL Capsule Number

Container Identification F-2

Set Identification:

Haye 4 of 4

\begin{tabular}{|c|c|c|c|c|c|c|c|c|c|c|}
\hline \multicolumn{8}{|c|}{ Container Identification $\quad \mathrm{F}-2$} & \multirow{2}{*}{\multicolumn{2}{|c|}{$\frac{\mathrm{R}:}{\text { itor Description }}$}} & \multirow{3}{*}{$\begin{array}{l}2:-36.58 \mathrm{~cm} \\
\text { Rellarks }\end{array}$} \\
\hline \multirow[b]{2}{*}{ Material } & \multirow{2}{*}{$\begin{array}{l}\text { Purchase Order } \\
\text { Number }\end{array}$} & \multirow{2}{*}{$\begin{array}{l}\text { Batch } \\
\text { Number }\end{array}$} & \multirow[b]{2}{*}{ Description } & \multirow{2}{*}{$\begin{array}{c}\text { Material } \\
\text { Weight } \\
\text { (ma) }\end{array}$} & \multicolumn{3}{|c|}{ Encapsulated Monitor Description } & & & \\
\hline & & & & & In & $\begin{array}{l}\text { Weight } \\
\text { (mqg) }\end{array}$ & $\begin{array}{l}\text { Diam } \\
\text { (in.) } \\
\end{array}$ & $\begin{array}{l}\text { Length } \\
\text { (in.) }\end{array}$ & Ident & \\
\hline $0.1 \%$ TaAl $(17)$ & 03783 & ORNL & 0.1 -in. $L \times 0.020$-in. 0 & 0.5660 & & & & & & \\
\hline $1.0 \% \operatorname{TaV}(17)$ & 32548 & ORNL & 0.1 -in. $L \times 0.020$-in. $D$ & 2.924 & & & & & & \\
\hline $\mathrm{Fe}(17)$ & 19047 & & $\begin{array}{l}\text { Metal Wire } \\
0.1-\text { in. } L \times 0.020-\text { in. } 0\end{array}$ & 3.738 & & & & & & \\
\hline${ }^{239} \mathrm{Pu}(18)$ & 14965 & $453-\mathrm{BO}$ & Oxide Wire & 1.280 & $\mathrm{v}$ & 51.235 & 0.050 & 0.340 & 34 & Series $x$ \\
\hline $\mathrm{Fe}(18)$ & 19046 & & $\begin{array}{l}\text { Metal Wire } \\
0.1-\text {-in. } L \times 0.020-\text { in. } 0\end{array}$ & 3.641 & & & & & & \\
\hline & & & & & & & & & & \\
\hline & & & & & & & & & & \\
\hline & & & & & & & & & & \\
\hline & & & & & & & & & & \\
\hline & & & & & & & & & & \\
\hline & & & & & & & & & & \\
\hline & & & & & & & & & & \\
\hline & & & & & & & & & & \\
\hline & & & & & & & & & & \\
\hline
\end{tabular}

离 
TABLE A-3

RUN 97, CONTAINER F-3, SPECTRAL SET DETAILS

Test: Run 97

Page ! of $\underline{2}$

HEDL Capsule Number

Container Identification F-3

\begin{tabular}{|c|c|c|c|c|c|c|c|c|c|c|}
\hline \multirow[b]{2}{*}{ Material } & \multirow[b]{2}{*}{$\begin{array}{c}\text { Purchase Order } \\
\text { Number }\end{array}$} & \multirow[b]{2}{*}{$\begin{array}{l}\text { Batch } \\
\text { Number }\end{array}$} & \multirow[b]{2}{*}{ Description } & \multirow{2}{*}{$\begin{array}{c}\text { Material } \\
\text { Height } \\
\text { (ing) }\end{array}$} & \multicolumn{5}{|c|}{ Encapsulated Monitor Description } & \multirow[b]{2}{*}{ Rewarks } \\
\hline & & & & & In & $\begin{array}{c}\text { Weight } \\
\text { (mg) }\end{array}$ & $\begin{array}{l}\text { Diam } \\
\text { (in.) }\end{array}$ & $\begin{array}{l}\text { Length } \\
\text { (in.) }\end{array}$ & Ident & \\
\hline${ }^{238} \mathrm{UO}_{2}(1)^{*}$ & 39407 & ES-2 & Oxide Wire & 7.283 & $v$ & 51.225 & 0.050 & 0.310 & $28:$ & Series i \\
\hline Nb (1) & Fabry & Kaweck i & $0.47 \mathrm{~mm} \times 0.3 \mathrm{~mm}$ & 4.560 & $v$ & 20.54 & 0.035 & 0.240 & 0 & \\
\hline $\mathrm{Ni}(1)$ & 1 & & $\begin{array}{l}\text { Wire } \\
0.1-i n . L \times 0.020-\text { in. } D\end{array}$ & 4.228 & & & & & & \\
\hline $2350(2)$ & 55047 & $264-C$ & Oxide Wire & 1.533 & $v$ & 21.574 & 0.050 & 0.125 & $24::$ & $\beta$ \\
\hline $23^{3} \mathrm{~Np} \mathrm{(3)}$ & 39407 & $N p-24-H p$ & Oxide Wire & 5.486 & $v$ & 51.081 & 0.050 & 0.350 & 16 & Beries I \\
\hline $\mathrm{Ti}(3)$ & 69926 & & $\begin{array}{l}\text { Wire } \\
0.1 \text {-in. } L \times 0.020-\text { in. } D\end{array}$ & 2.133 & & & & & & \\
\hline Mn (4) & 55086 & & $\begin{array}{l}0 \times \text { ide Wire } \\
0.125-\text { in. } L \times 0.020 \text {-in. } D\end{array}$ & 0.93684 & v & 17.437 & 0.035 & 0.240 & $F$ & \\
\hline SS wire (4) & & & 0.1 -in. $L \times 0.033$-in. $D$ & 9.872 & & & & & & FOTA SS Rod \\
\hline $\mathrm{SC}_{2} \mathrm{O}_{3}$ (5) & 39407 & ORNL-1 & Oxide Wire & 1.298 & lv & 40.855 & 0.050 & 0.250 & $87: \ldots$ & \\
\hline$\left[\begin{array}{l}0.16 \mathrm{Sc}_{2} \mathrm{O}_{3} / \mathrm{MgO} \\
(6)^{2}\end{array}\right.$ & 55046 & 1 & $\begin{array}{l}\text { 0xide Wire } \\
0.125-\text { in. } L \times 0.020 \text {-in. } D\end{array}$ & 0.7649 & v & 20.10 & 0.035 & 0.300 & 3 & \\
\hline $0.1 \%$ AuAl (6) & & Rx Wire & Metal Wire & 1.3116 & v & 14.544 & 0.035 & 0.180 & $\&$ & \\
\hline $1.0 \% \operatorname{cov}(7)$ & Fabry & $S p-2440$ & $\begin{array}{l}\text { Metal Wire } \\
0.100-\text { in. L } \times 0.015-\text { in. D }\end{array}$ & 2.006 & Iv & 16.544 & 0.035 & 0.270 & H & \\
\hline$\left(\begin{array}{l}0.1 \% \\
(7)\end{array}\right.$ & 27940 & JORNL Tube 1 & $\begin{array}{l}\text { Oxide Wire } \\
0.100-i n . L \times 0.020-\text { in. } D\end{array}$ & 1.299 & lv & 25.56 & 0.035 & 0.300 & $z$ & Series 2 \\
\hline $0.1 \% \operatorname{COAI}(7)$ & HA-69-889-085 & SRM 953 & $\begin{array}{l}\text { Metal wire } \\
0.100-i n . L \times 0.020-i n . D\end{array}$ & 1.2518 & IV & 18.259 & 0.035 & 0.240 & 1 & \\
\hline
\end{tabular}

*Number in parentheses $=$ hole number in container 
TABLE A-3 (Continued)

Test: Run 97

Page $\underline{2}$ of $\underline{2}$

HEDL Capsule Number

Container Identification $\mathrm{F}-3$

Set Identification:

R:

$2:-15.24 \mathrm{~cm}$

\begin{tabular}{|c|c|c|c|c|c|c|c|c|c|c|}
\hline \multirow[b]{2}{*}{ Material } & \multirow[b]{2}{*}{$\begin{array}{l}\text { Purchase Order } \\
\text { Number }\end{array}$} & \multirow[b]{2}{*}{$\begin{array}{l}\text { Batch } \\
\text { Number }\end{array}$} & \multirow[b]{2}{*}{ Description } & \multirow{2}{*}{$\begin{array}{c}\text { Material } \\
\text { Weight } \\
\text { (mg) }\end{array}$} & \multicolumn{5}{|c|}{ Encapsulated Monitor Description } & \multirow[b]{2}{*}{ Remarks } \\
\hline & & & & & In & $\begin{array}{c}\text { Weight } \\
(\mathrm{mg})\end{array}$ & $\begin{array}{c}\text { Diam } \\
\text { (in.) }\end{array}$ & $\begin{array}{c}\text { Length } \\
\text { (in.) }\end{array}$ & Ident & \\
\hline $0.1 \%$ AgAl (8) & 03783 & Rx Wire & Metal Wire & 1.2962 & v & 15.057 & 0.035 & 0.210 & 6 & \\
\hline${ }^{2}{ }^{9} \mathrm{Pu}_{\mathrm{u}}$ (9) & 39407 & $453-B 0$ & Oxide Wire & 1.372 & v & 44.921 & 0.050 & 0.280 & 5 & \\
\hline $0.1 \% \operatorname{TaAT}(9)$ & 03783 & $217 \mathrm{~W}$ & $\begin{array}{l}\text { Metal Wire } \\
0.100-i n . L \times 0.020-i n .0\end{array}$ & 0.7025 & v & 17.336 & 0.035 & 0.240 & $T$ & \\
\hline $1.0 \% \operatorname{TaV}(9)$ & 32548 & & $\begin{array}{l}\text { Metal Wire } \\
0.100-i n . L \times 0.020-i n .0\end{array}$ & 2.889 & & & & & & \\
\hline $\mathrm{Fe}(10)$ & 19046 & Sc 53 & $\begin{array}{l}\text { Metal wire } \\
0.100-i n . L \times 0.020-i n .0\end{array}$ & 3.948 & v & 20.54 & 0.035 & 0.240 & $\mathrm{~s}$ & Series 10 \\
\hline $\mathrm{Fe}(10)$ & B65-777-19046 & & $\begin{array}{l}\text { Metal Wire } \\
0.100-i n . L \times 0.020-\text { in. } D\end{array}$ & 3.758 & & & & & & Rx Spool 3 \\
\hline${ }^{6} \operatorname{LiF}(11)$ & 44051 & AI & Crystal & 2.585 & v & 43.585 & 0.050 & 0.250 & L201-HF & \\
\hline${ }^{10} \mathrm{~B}(12)$ & 44051 & AI & Crystal & 1.235 & v & 41.862 & 0.050 & 0.250 & B245-IL & \\
\hline $\mathrm{Nb}(13)$ & Fabry & Kaweck i & $\begin{array}{l}\text { Metal Wire } \\
0.47 \mathrm{~mm} \times 0.3 \mathrm{~mm}\end{array}$ & 4.571 & & & & & 84 & \\
\hline $\mathrm{Cu}(13)$ & 19047 & & $\begin{array}{l}\text { Wire } \\
0.100-i n . L \times 0.020-i n .0\end{array}$ & 4.083 & & & & & & $\begin{array}{l}\text { Cominco Americar } \\
\text { Roll } 1\end{array}$ \\
\hline & & & & & & & & & & \\
\hline & & & & & & & & & & \\
\hline & & & & & & & & & & \\
\hline & & & & & & & & & & \\
\hline
\end{tabular}


TABLE $A-4$

RUN 97, CONTAINER HWW-1, SPECTRAL SET DETAILS

Test: Bun 27

HEDL Capsule Number

Container Identification Spectral Set

Set Identification:

HWW-1

$R$ :

Fage 1 of 1

\begin{tabular}{|c|c|c|c|c|c|c|c|c|c|c|}
\hline \multirow[b]{2}{*}{ Material } & \multirow[b]{2}{*}{$\begin{array}{c}\text { Purchase Order } \\
\text { Number }\end{array}$} & \multirow[b]{2}{*}{$\begin{array}{l}\text { Batch } \\
\text { Number }\end{array}$} & \multirow[b]{2}{*}{ Description } & \multirow{2}{*}{$\begin{array}{c}\begin{array}{c}\text { Material } \\
\text { Weight } \\
\text { (mg) }\end{array} \\
\end{array}$} & \multicolumn{5}{|c|}{ Encapsulated Monitor Description } & \multirow[b]{2}{*}{ Remarks } \\
\hline & & & & & $\ln$ & $\begin{array}{c}\text { Weight } \\
\text { (mig) }\end{array}$ & $\begin{array}{l}\text { Diam } \\
\text { (in.) }\end{array}$ & $\begin{array}{l}\text { Length } \\
\text { (in.) }\end{array}$ & Ident & \\
\hline $235 U$ & 39462 & $264-c$ & Oxide Wire & 1.519 & v & 17.073 & 0.035 & 0.190 & 0 & Series 2 \\
\hline${ }^{238} \mathrm{U}$ & 32542 & $E S-Z$ & Oxide Wire & 8.337 & $v$ & 25.780 & 0.035 & 0.315 & 2 & \\
\hline${ }^{237} \mathrm{~Np}$ & 39462 & $\mathrm{~Np}-24-\mathrm{Hp}$ & Oxide Wire & 3.258 & $v$ & 23.277 & 0.035 & 0.280 & $\mathrm{H}$ & Series 2 \\
\hline $\mathrm{Sc}_{2} \mathrm{O}_{3}$ & 39462 & ORNL-1 & Oxide Wire & 1.334 & $v$ & 25.102 & 0.035 & 0.340 & $s$ & Series 1 \\
\hline $0.1 \% \operatorname{COA} 1$ & HA-69-889.050 & SRM 953 & $\begin{array}{l}\text { Metal Wire } \\
0.1 \text {-in. L } \times 0.020 \text {-in. D }\end{array}$ & 1.2746 & v & 17.150 & 0.035 & 0.240 & 3 & \\
\hline $239 \mathrm{Pu}$ & 39462 & $453-B 0$ & exide hire & 1.542 & w & 21.654 & 0.035 & 0.250 & $N$ & Series 6 \\
\hline $\mathrm{Fe}$ & 19046 & Sc 53 & $\begin{array}{l}\text { Metal Wire } \\
0.100-i n .1 \times 0.020-\text { in. } D\end{array}$ & 4.009 & $v$ & 19.709 & 0.035 & 0.240 & 3 & Series 5 \\
\hline${ }^{6} \mathrm{LiF}$ & 50070 & AI & Crystal & 0.0702 & $v$ & 18.936 & 0.035 & 0.250 & LB142-H9 & \\
\hline SS & & & 0.7 -in. $L \times 0.033$-in. $D$ & 10.851 & & & & & & FOTA SS ROd \\
\hline${ }^{10} B$ & 50070 & AI & Crystal & 0.0670 & $v$ & 18.493 & 0.035 & 0.250 & BB163-1X & \\
\hline $\mathrm{Ni}$ & 1 & & $\begin{array}{l}\text { Metal Wire } \\
0.1-\text { in. L } \quad \text { x } 0.020-\text { in. D }\end{array}$ & 4.399 & v & 17.95 & 0.035 & 0.180 & 6 & Series 3 \\
\hline $0.7 \%$ CoMg0 & 27940 & ORNL-1 & $\begin{array}{l}\text { Metal Wire } \\
0.1 \text {-in.L } \quad \text { x } 0.020-\text { in. } 0\end{array}$ & 1.102 & Iv & 20.70 & 0.035 & 0.300 & 2 & Series 3 \\
\hline $\mathrm{Ii}$ & 69925 & $139-W$ & $\begin{array}{l}\text { Metal Wire } \\
0.1 \text {-in. } . \times 0.020-\text { in. } 0\end{array}$ & 2.033 & v & 20.53 & 0.035 & 0.240 & $x$ & Series 2 \\
\hline $\mathrm{Cu}$ & 19047 & CPO-3054 & $\begin{array}{l}\text { Metal Wire } \\
0.1 \text {-in. } L \times 0.020 \text {-in. D }\end{array}$ & 4.069 & Iv & 20.14 & 0.035 & 0.210 & 4 & Series 2 \\
\hline
\end{tabular}

See Table 6 for Loading Order 
TABLE $A-5$

RUN 97, CONTAINER F-4, SPECTRAL SET DETAILS

Test: Run 97

Page $1 .$. of 4

HEDL Capsule Number

Container Identification $\mathrm{F}-4$ Set Identification:

\begin{tabular}{|c|c|c|c|c|c|c|c|c|c|c|}
\hline Material & $\begin{array}{c}\text { Purchase Order } \\
\text { Number }\end{array}$ & $\begin{array}{l}\text { Batch } \\
\text { Number }\end{array}$ & Description & $\begin{array}{c}\text { Material } \\
\text { We ight } \\
\text { (mg) }\end{array}$ & $\ln$ & $\begin{array}{l}\text { Weight } \\
(\mathrm{mg})\end{array}$ & $\begin{array}{l}\text { Diam } \\
\text { (in.) }\end{array}$ & $\begin{array}{c}\text { Length } \\
\text { (in.) }\end{array}$ & Ident & Remlarks \\
\hline${ }^{235} U(1) *$ & 55047 & $264-C$ & Oxide Wire & 1.443 & v & 20.869 & 0.050 & 0.125 & $31 \quad \vdots$ & Series 3 \\
\hline $23{ }^{2} \mathrm{U}(1)$ & 39407 & ES-Z & 0xide Wire & 7.498 & $v$ & 51.305 & 0.050 & 0.315 & 34 & Series I \\
\hline $2{ }^{2}{ }^{7} \mathrm{NpO}_{2}$ & 39407 & Np-24-Hp & Oxide Wire & 5.907 & $v$ & 49.894 & 0.050 & 0.345 & 17 & Series I \\
\hline $1.0 \% \operatorname{Cov}(1)$ & Fabry & Sp-2440 & $\begin{array}{l}\text { Metal Wire } \\
0.1-\text { in. } L \times 0.015-i n .0\end{array}$ & 1.9301 & & & & & & \\
\hline $\mathrm{Nb}(1)$ & Fabry & Kawecki & $0.47 \mathrm{~mm} \times 3.0 \mathrm{~mm}$ & 4.577 & & & & & 86 & \\
\hline $\mathrm{Fe}(1)$ & B65-777-19046 & & $\begin{array}{l}\text { Wire } \\
0.100-\text { in. } L \times 0.020-\text { in. D }\end{array}$ & 4.000 & & & & & & \\
\hline $\mathrm{Ni}(1)$ & 1 & & $\begin{array}{l}\text { Wire } \\
0.100-\text { in. } L \times 0.020 \text {-in. D }\end{array}$ & 4.305 & & & & & & \\
\hline $\mathrm{Cu}(1)$ & 19047 & & $\begin{array}{l}\text { Wire } \\
0.100-i n . L \times 0.020-i n . ~ D\end{array}$ & 3.987 & & & & & & $\begin{array}{l}\text { Cominco American } \\
\text { Rolli }\end{array}$ \\
\hline Ti (1) & 69926 & & $\begin{array}{l}\text { Wire } \\
0.100-i n . L \times 0.020-i n .0\end{array}$ & 2.146 & & & & & & \\
\hline${ }^{238} \mathrm{UO}_{2}$ (2) & 32542 & ES-Z & Oxide Wire & 8.696 & $v$ & 26.915 & 0.035 & 0.310 & 3 & \\
\hline $\mathrm{Nb}(2)$ & Fabry & Kawecki & $0.47 \mathrm{~mm} \times 3.0 \mathrm{~mm}$ & 4.560 & & & & & 80 & \\
\hline $235 \cup(3)$ & 55047 & $264-C$ & Oxide Wire & 1.446 & $v$ & 20.853 & 0.050 & 0.125 & 33 & Series 3 \\
\hline $235 \mathrm{U}(3)$ & 39462 & $264-C$ & Oxide Wire & 1.529 & $v$ & 17.491 & 0.035 & 0.190 & 2 & Series 2 \\
\hline${ }^{10} \mathrm{~B} \quad(3)$ & 44051 & AI & Crystal & 0.864 & Iv & 40.118 & 0.050 & 0.250 & B24-P5 & \\
\hline
\end{tabular}

* Number in parentheses $=$ hole number in container . 
TABLE A-5 (Continued)

Test: Run 97

HEDL Capsule Number

Container Identification

$F-4$

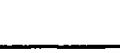

Set Identification:

Page 2 of 4

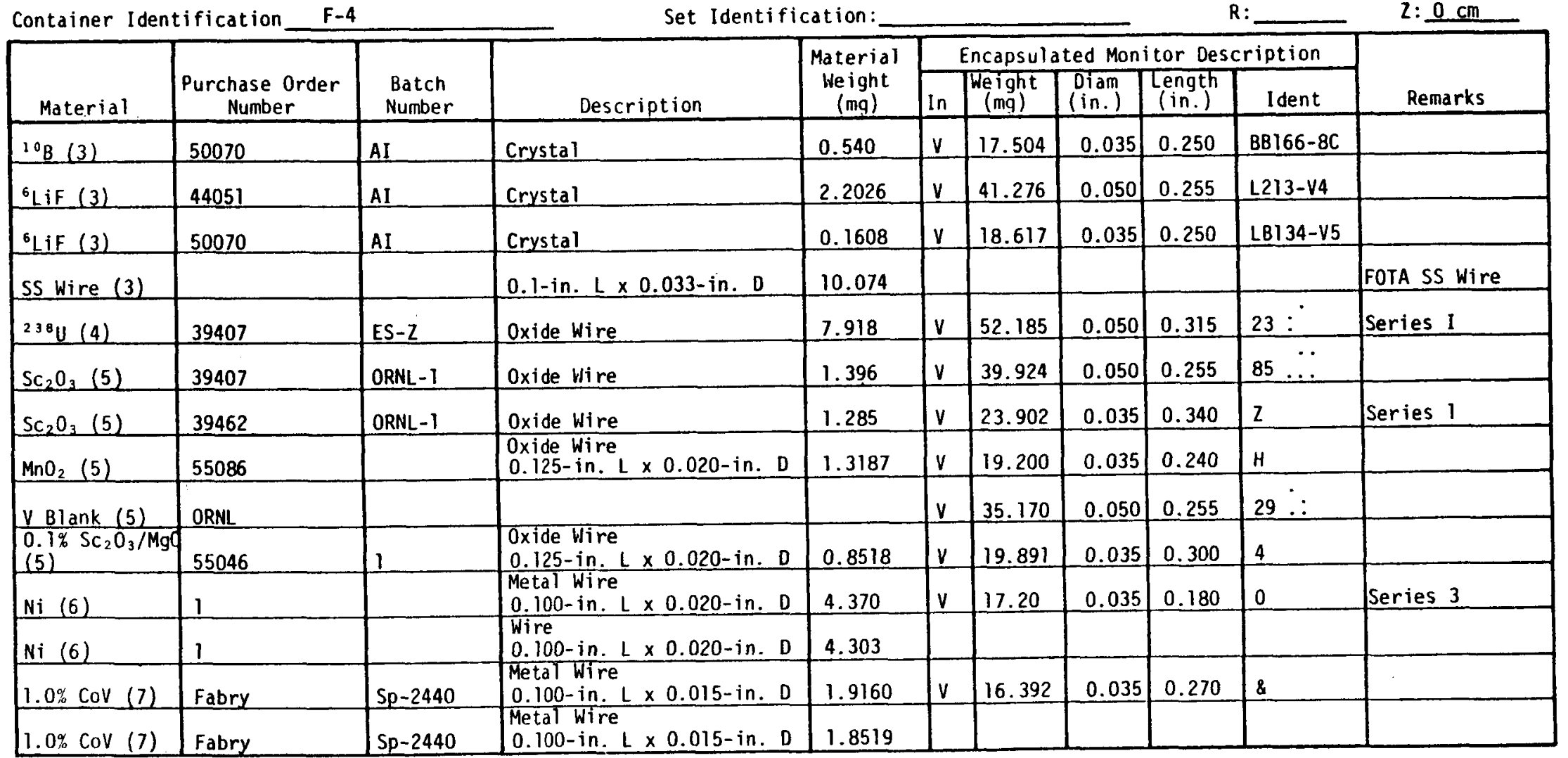


TABLE A-5 (Continued)

Test: Run 97

HEOL Capsule Number

Container Identification F-4

Set Identification:

Page 3. of 4

\begin{tabular}{|c|c|c|c|c|c|c|c|c|c|c|}
\hline \multicolumn{3}{|l|}{ Container Id } & \multicolumn{5}{|c|}{ Set Identification: } & \multicolumn{2}{|c|}{$R:=$} & \multirow[t]{3}{*}{$2: \underline{0 \mathrm{~cm}}$} \\
\hline \multirow[b]{2}{*}{ Material } & \multirow[b]{2}{*}{$\begin{array}{c}\text { Purchase Order } \\
\text { Number }\end{array}$} & \multirow[b]{2}{*}{$\begin{array}{l}\text { Batch } \\
\text { Number }\end{array}$} & \multirow[b]{2}{*}{ Description } & \multirow{2}{*}{$\begin{array}{c}\text { Material } \\
\text { Weight } \\
\text { (mg) }\end{array}$} & \multicolumn{5}{|c|}{ Encapsulated Monitor Description } & \\
\hline & & & & & In & $\begin{array}{c}\text { Weight } \\
\text { (mg) }\end{array}$ & $\begin{array}{c}\text { Diain } \\
\text { (in.) }\end{array}$ & $\begin{array}{l}\text { Length } \\
\text { (in.) }\end{array}$ & Ident & \\
\hline $0.1 \%$ CoMg0 (7) & 27940 & ORNL Tube 1 & $\begin{array}{l}\text { Uxide WTre } \\
0.100 \text {-in. } L \times 0.020 \text {-in. } D\end{array}$ & 1.349 & $V$ & 25.82 & 0.035 & 0.300 & $T$ & Series 2 \\
\hline $0.1 \% \operatorname{COAl}(7)$ & $H A-69-889-085$ & SRM 953 & $\begin{array}{l}\text { Metal Wire } \\
0.100-\text { in. } L \times 0.020 \text {-in. D }\end{array}$ & 1.2650 & V & 17.860 & 0.035 & 0.240 & 2 & \\
\hline $\mathrm{Cu}(8)$ & 19047 & CPO 3054 & $\begin{array}{l}\text { Metal Wire } \\
0.100-i n . L \times 0.020-\text { in. } D\end{array}$ & 4.142 & 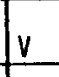 & 18.60 & 0.035 & 0.210 & 6 & Series 2 \\
\hline $\mathrm{Cu}(8)$ & 19047 & & $\begin{array}{l}\text { Wire } \\
0.100-\text { in. L } \times 0.020 \text {-in. D }\end{array}$ & 4.100 & & & & & & \\
\hline $237 \mathrm{ND}(\mathrm{g})$ & 39402 & $\mathrm{No}-24-\mathrm{HR}$ & Oxide Wire & 5.840 & ty & 52.083 & 0.050 & 0.345 & $18^{\cdots}$ & Series L \\
\hline $23^{7} \mathrm{~Np}(9)$ & 39462 & $\mathrm{Hp}-24-\mathrm{Hp}$ & Oxide Wire & 3,199 & fr & 23.000 & 0.035 & 0.280 & $x$ & Series 2 \\
\hline $239 \mathrm{Pu}(9)$ & 39407 & $453-80$ & Oxide Wire & 1.625 & $v$ & 48.490 & 0.050 & 0.285 & 1. & \\
\hline${ }^{239} \mathrm{Pu} \quad(9)$ & 39462 & 453-B0 & Oxide Wire & 1.257 & v & 21.264 & 0.035 & 0.250 & J & Series 6 \\
\hline $\mathrm{Fe}(9)$ & 19046 & Sc 53 & $\begin{array}{l}\text { Metal Wire } \\
0.100-\text { in. } L \times 0.020-\text { in. } D\end{array}$ & 4.025 & v & 20.74 & 0.035 & 0.240 & v & Series 10 \\
\hline $\mathrm{Fe}(9)$ & B65-777-19046 & & $\begin{array}{l}\text { Wire } \\
0.100-\text { in. } L \times 0.020-\text { in. } D\end{array}$ & 3.808 & & & & & & \\
\hline $\mathrm{Ti}(10)$ & 69925 & $139 W$ & $\begin{array}{l}\text { Metal Wire } \\
0.100-i n . L \times 0.020-i n . D\end{array}$ & 2.162 & $y$ & 18.71 & 0.035 & 0.280 & $k$ & Series 3 \\
\hline $\mathrm{Ti}(10)$ & 69926 & & $\begin{array}{l}\text { Wire } \\
0.100-\text { in. L } \times 0.020-\text { in. } D\end{array}$ & 2.143 & & & & & & \\
\hline $1.0 \% \operatorname{TaV}(10)$ & 32548 & ORNL & 0.100 -in. $L \times 0.020$-in. D & 2.995 & & & & & & \\
\hline $\begin{array}{l}0.1 \% \mathrm{TaAl} \\
\text { (ii) }\end{array}$ & 03783 & $217 \mathrm{~W}$ & $\begin{array}{l}\text { Metal Wire } \\
0.100-\text { in. } L \times 0.020-\text { in. } 0\end{array}$ & 0.7000 & IV & 17.059 & 0.035 & 0.240 & $x$ & Bx Roll 1 \\
\hline
\end{tabular}


TABLE A-5 (Continued)

Test: Run 97

HEDL Capsule Number

Container Identification

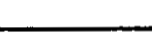

\begin{tabular}{|c|c|c|c|c|c|c|c|c|c|c|}
\hline \multirow[b]{2}{*}{ Material } & \multirow[b]{2}{*}{$\begin{array}{l}\text { Purchase Order } \\
\text { Number }\end{array}$} & \multirow[b]{2}{*}{$\begin{array}{l}\text { Batch } \\
\text { Number }\end{array}$} & \multirow[b]{2}{*}{ Description } & \multirow{2}{*}{$\begin{array}{c}\text { Material } \\
\text { Weight } \\
\text { (ma) }\end{array}$} & \multicolumn{5}{|c|}{ Encapsulated Monitor Description } & \multirow[b]{2}{*}{ Remarks } \\
\hline & & & & & In & \begin{tabular}{|l|} 
Weight \\
$(\mathrm{mg})$
\end{tabular} & \begin{tabular}{|l|} 
Diam \\
(in.) \\
\end{tabular} & \begin{tabular}{|l} 
Length \\
(in.) \\
\end{tabular} & Ident & \\
\hline $0.1 \%$ TaAl (11) & 03783 & $217 \mathrm{~W}$ & $\begin{array}{l}\text { Tetar wire } \\
0.100 \text {-in. } L \times 0.020 \text {-in. } 0\end{array}$ & 0.7000 & & & & & & Rx Roll I \\
\hline $0.1 \%$ AUA] (12) & & Rx Hire & Metal Wire & 1.3267 & v & 14.768 & 0.035 & 0.180 & N & \\
\hline $0.16 \mathrm{AgAl} \quad(13)$ & 03783 & Rx Hire & Metal Wire & 1.1744 & v & 15.821 & 0.035 & 0.210 & 7 & \\
\hline & & & & & & & & & & \\
\hline & & & & & & & & & & \\
\hline & & & & & & & & & & \\
\hline & & & & & & & & & & \\
\hline & & & & & & & & & & \\
\hline & & & & & & & & & & \\
\hline & & & & & & & & & & \\
\hline & & & & & & & & & & \\
\hline & & & & & & & & & & \\
\hline & & & & & & & & & & \\
\hline & & & & & & & & & & \\
\hline
\end{tabular}

Page 4 of 4 
TABLE $A-6$

RUN 97, CONTAINER F-5, SPECTRAL SET DETAILS

Test: Run 97

HEDL Capsule Number

Container Identification

$F-5$

Set Identification :

$R$ :

Page 1 of 2

\begin{tabular}{|c|c|c|c|c|c|c|c|c|c|c|}
\hline \multirow[b]{2}{*}{ Material } & \multirow[b]{2}{*}{$\begin{array}{c}\text { Purchase Order } \\
\text { Number }\end{array}$} & \multirow[b]{2}{*}{$\begin{array}{l}\text { Batch } \\
\text { Number }\end{array}$} & \multirow[b]{2}{*}{ Description } & \multirow{2}{*}{$\begin{array}{c}\text { Material } \\
\text { Weight } \\
\text { (mq) }\end{array}$} & \multicolumn{5}{|c|}{ Encapsulated Monitor Description } & \multirow[b]{2}{*}{ Remarks } \\
\hline & & & & & In & $\begin{array}{c}\text { We ight } \\
\text { (mig) }\end{array}$ & $\begin{array}{l}\text { Diain } \\
\text { (in.) }\end{array}$ & $\begin{array}{l}\text { Length } \\
\text { (in.) }\end{array}$ & Ident & \\
\hline${ }^{23 \theta} U(1)^{*}$ & 39407 & ES-Z & 0xide Wire & 7.556 & $v$ & 51.455 & 0.050 & 0.315 & $9::$ & Series I \\
\hline $\mathrm{Nb}(1)$ & Fabry & Kaweck i & $0.47 \mathrm{~mm} \times 3.0 \mathrm{~mm}$ & 4.567 & $v$ & 20.37 & 0.035 & 0.240 & 1 & \\
\hline $\mathrm{Ni}$ (1) & 1 & & $\begin{array}{l}\text { Wire } \\
0.100-\text { in. } L \times 0.020 \text {-in. } 0\end{array}$ & 4.399 & & & & & & \\
\hline${ }^{235} \mathrm{U}(2)$ & 55047 & $264-C$ & Oxide Wire & 1.535 & v & 20.909 & 0.050 & 0.125 & 45 & Series 3 \\
\hline $2^{27} \mathrm{~Np} \mathrm{(3)}$ & 39407 & $\mathrm{~Np}-24-\mathrm{Hp}$ & Oxide Wire & 5.800 & v & 50.598 & 0.050 & 0.345 & 20 & Series 1 \\
\hline $\mathrm{Ti}$ (3) & 69926 & & $\begin{array}{l}\text { Wire } \\
0.100-i n . L \times 0.020-i n . D\end{array}$ & 2.124 & & & & & & \\
\hline $\mathrm{Mn}_{3} \mathrm{O}_{2} \quad(4)$ & 55086 & & $\begin{array}{l}\text { Oxide Wire } \\
0.125-i n . L \times 0.020-i n .0 \\
\end{array}$ & 1.2426 & V & 17.617 & 0.035 & 0.240 & 5 & \\
\hline SS Wire (4) & & & $0.100-$ in. $L \times 0.033-i n . D$ & 9.277 & & & & & & FOTA SS Wire \\
\hline $\mathrm{Sc}_{2} \mathrm{O}_{3}$ (5) & 39407 & \begin{tabular}{|l|} 
ORNL \\
Batch 1 \\
\end{tabular} & Oxide Wire & 1.377 & v & 41.165 & 0.050 & 0.255 & 89 & \\
\hline $\begin{array}{l}0.1 \% \mathrm{Sc}_{2} \mathrm{O}_{3} / \mathrm{MgC} \\
(5)^{-}\end{array}$ & 55046 & S a cin & $\begin{array}{l}\text { Oxide Wire } \\
0.125-i n . L \times 0.020-i n .0\end{array}$ & 0.8714 & $V$ & 19.481 & 0.035 & 0.300 & 2 & \\
\hline $0.1 \%$ AuAl (6) & & & $\begin{array}{l}\text { Metal Wire } \\
0.100-\text { in. } L \times 0.020-\text { in. } D\end{array}$ & 1.2260 & $v$ & 14.906 & 0.035 & 0.180 & $J$ & \\
\hline $1.0 \% \operatorname{CoV}(7)$ & Fabry & Sp-2440 & $\begin{array}{l}\text { Metal Wire } \\
0.100-\text { in. } L \times 0.015-i n . D\end{array}$ & 1.9970 & v & 17.410 & 0.035 & 0.180 & $F$ & \\
\hline $0.1 \%$ CoMgO (7) & 27940 & ORNL Tube 1 & $\begin{array}{l}\text { Oxide Wire } \\
0.100 \text { - in. } L \times 0.020 \text {-in. } D\end{array}$ & 1.165 & v & 20.90 & 0.035 & 0.300 & 6 & Series 3 \\
\hline $0.1 \% \operatorname{COAI}(7)$ & HA- $69-889-085$ & SRM 953 & $\begin{array}{l}\text { Meta Wire } \\
0.100-\text { in. L } \times 0.020-i n .0\end{array}$ & 1.2850 & Iv & 17.817 & 0.035 & 0.240 & 10 & \\
\hline
\end{tabular}

*Number in parentheses $=$ hole number in container. 
TABLE A-6 (Continued)

Test: Run 97

HEDL Capsule Number

Container Identification F-5
Page 2 of $\underline{2}$

Set Identification:

\begin{tabular}{|c|c|c|c|c|c|c|}
\hline \multirow{2}{*}{$\begin{array}{c}\text { Material } \\
\text { We ight } \\
\text { (mg) }\end{array}$} & \multicolumn{5}{|c|}{ Encapsulated Monitor Description } & \multirow[b]{2}{*}{ Renliarks } \\
\hline & In & $\begin{array}{c}\text { Weight } \\
\text { (mo) }\end{array}$ & $\begin{array}{l}\text { Diam } \\
\text { (in.) }\end{array}$ & $\begin{array}{c}\text { Length } \\
\text { (in.) }\end{array}$ & Ident & \\
\hline 1.3718 & v & 16.517 & 0.035 & 0.210 & 3 & \\
\hline 1.108 & v & 49.676 & 0.050 & 0.285 & $12 \ldots$ & Series $x$ \\
\hline 0.7505 & $\underline{v}$ & 17.261 & 0.035 & 0.240 & v & \\
\hline \multicolumn{7}{|l|}{2.816} \\
\hline 3.828 & $\underline{v}$ & 20.10 & 0.035 & 0.240 & 4 & Series 10 \\
\hline \multicolumn{7}{|l|}{3.882} \\
\hline 2.144 & $v$ & 42.796 & 0.050 & 0.255 & $L 211-A 2$ & \\
\hline 1.110 & $v$ & 42.605 & 0.050 & 0.255 & B244-F5 & \\
\hline \multicolumn{7}{|l|}{4.572} \\
\hline \multicolumn{7}{|l|}{4.106} \\
\hline & & & & & & \\
\hline & & & & & & \\
\hline & & & & & & \\
\hline & & & & & & \\
\hline
\end{tabular}


TABLE A-7

RUN 97, CONTAINER F-6, SPECTRAL SET DETAILS

Test: __ Run 97

HEDL Capsule Number

Container Identification

$F-6$

Set Identification:

$\quad 2:+39.37 \mathrm{~cm}$

\begin{tabular}{|c|c|c|c|c|c|c|c|c|c|c|}
\hline \multirow[b]{2}{*}{ Material } & \multirow[b]{2}{*}{$\begin{array}{l}\text { Purchase Order } \\
\text { Number }\end{array}$} & \multirow[b]{2}{*}{$\begin{array}{l}\text { Batch } \\
\text { Number }\end{array}$} & \multirow[b]{2}{*}{ Description } & \multirow{2}{*}{$\begin{array}{c}\text { Material } \\
\text { Weight } \\
\text { (mg) }\end{array}$} & \multicolumn{5}{|c|}{ Encapsulated Monitor Description } & \multirow[b]{2}{*}{ Remarks } \\
\hline & & & & & In & $\begin{array}{c}\text { Weight } \\
\text { (mg) }\end{array}$ & $\begin{array}{l}\text { Diam } \\
\text { (in.) }\end{array}$ & $\begin{array}{l}\text { Length } \\
\text { (in.) }\end{array}$ & Ident & \\
\hline $1.0 \% \operatorname{cov}(1) *$ & Fabry & & $\begin{array}{l}\text { Meta Wire } \\
0.1 \text {-in. L } 0.015 \text {-in. D }\end{array}$ & 1.8333 & & & & & & \\
\hline $\mathrm{Ni}(1)$ & 1 & & $\begin{array}{l}\text { Meta Wire } \\
0.1-i n . L \times 0.020-i n . D\end{array}$ & 4.401 & & & & & & \\
\hline $\mathrm{Fe}(1)$ & 19046 & & $\begin{array}{l}\text { Metal Wire } \\
0.1-i n . L \times 0.020-i n .0\end{array}$ & 3.809 & & & & & & \\
\hline $\mathrm{Cu}(1)$ & 19047 & & $\begin{array}{l}\text { Metal Wire } \\
0.1-i n . L \times 0.020-i n .0\end{array}$ & 4.089 & & & & & & \\
\hline $\mathrm{Ti}(1)$ & 69926 & & $\begin{array}{l}\text { Wire } \\
0.1-i n . L \times 0.020-i n .0\end{array}$ & 2.151 & & & & & & \\
\hline $\mathrm{Fe}(2)$ & 19046 & & $0.1-i n . L \times 0.020-i n . D$ & 4.073 & & & & & & \\
\hline $\mathrm{Ni} \mathrm{(2)}$ & 1 & & $\begin{array}{l}\text { Metal Wire } \\
0.1-\text { in. L } \times 0.020 \text {-in. D }\end{array}$ & 4.380 & & & & & & \\
\hline $238 \mathrm{U}(3)$ & 39407 & ES- $Z$ & Oxide Wire & 7.354 & v & 52.506 & 0.050 & 0.315 & $29 \therefore$ & Series I \\
\hline $235 U(4)$ & 55047 & $264-C$ & Oxide Wire & 1.227 & v & 21.226 & 0.050 & 0.125 & $56 \ldots$ & Series 3 \\
\hline $2{ }^{37} \mathrm{~Np}(5)$ & 39407 & Np-24-Hp & Oxide Wire & 6.438 & v & 52.947 & 0.050 & 0.345 & $23::^{\circ}$ & Series I \\
\hline${ }^{2}{ }^{39} \mathrm{Pu}(6)$ & 14965 & 453-80 & Oxide Wire & 1.016 & v & 54.063 & 0.050 & 0.285 & $14:$ & Series $x$ \\
\hline $\mathrm{Sc}_{2} \mathrm{O}_{3}(7)$ & 39407 & PRNL-1 & Oxide Wire & 1.311 & $v$ & 42.547 & 0.050 & 0.255 & $92 \therefore$ & \\
\hline $\begin{array}{l}0.1 \% \mathrm{SC}_{2} \mathrm{O}_{3} / \mathrm{MgO} \\
(8)\end{array}$ & 55046 & 1 & $\begin{array}{l}\text { 0xide Wire } \\
0.125-i n . L \times 0.020-i n .0\end{array}$ & 0.9603 & lv & 20.04 & 0.035 & 0.300 & 7 & \\
\hline${ }^{10} \mathrm{~B}(9)$ & 44051 & AI & Crystal & 0.737 & lv & 41.342 & 0.050 & 0.255 & $8250-C 7$ & \\
\hline
\end{tabular}

*Number in parentheses $=$ hole number in container. 
TABLE A-7 (Continued)

Test: Run 97

Paye 2. of 2

HEDL Capsule Number

Container Identification

$-6$

Set Identification:

R:

$z:+39.37 \mathrm{~cm}$

\begin{tabular}{|c|c|c|c|c|c|c|c|c|c|c|}
\hline \multirow[b]{2}{*}{ Material } & \multirow[b]{2}{*}{$\begin{array}{c}\text { Purchase Order } \\
\text { Number }\end{array}$} & \multirow[b]{2}{*}{$\begin{array}{l}\text { Batch } \\
\text { Number }\end{array}$} & \multirow[b]{2}{*}{ Description } & \multirow{2}{*}{$\begin{array}{c}\begin{array}{c}\text { Material } \\
\text { We ight } \\
\text { (mg) }\end{array} \\
\end{array}$} & \multicolumn{5}{|c|}{ Encapsulated Monitor Description } & \multirow[b]{2}{*}{ Remarks } \\
\hline & & & & & In & \begin{tabular}{|c|} 
Weight \\
(mg)
\end{tabular} & $\begin{array}{l}\text { Diam } \\
\text { (in.) } \\
\end{array}$ & $\begin{array}{l}\text { Length } \\
\text { (in.) } \\
\end{array}$ & Ident & \\
\hline${ }^{6}$ LiF $(10)$ & 44051 & AI & Crystal & 1.892 & v & 43.517 & 0.050 & 0.255 & $\llcorner 214-14$ & \\
\hline $1.0 \% \operatorname{cov}(11)$ & Fabry & & $\begin{array}{l}\text { Metal Wire } \\
0.1-\text {-in. L } ~\end{array}$ & 1.8110 & & & & & & \\
\hline $\begin{array}{l}0.1 \% \text { CoMgO } \\
\text { (ii) }\end{array}$ & 27940 & ORNL Tube I & $\begin{array}{l}0 x i d e \text { Wire } \\
0.1 \text { - in. } L \times 0.020-\text { in. } D\end{array}$ & 1.226 & $\mathrm{v}$ & 20.45 & 0.035 & 0.300 & $\mathrm{~J}$ & Series 3 \\
\hline $0.1 \% \operatorname{COAI}(11)$ & & SRM-953 & $\begin{array}{l}\text { Metal Wire } \\
0.100-\text { in. } L \times 0.020-\text { in. } D\end{array}$ & 1.2683 & & & & & & \\
\hline $1.0 \% \operatorname{cov}(12)$ & Fabry & & $\begin{array}{l}\text { Metal Hire } \\
0.1 \text {-in. L } 0.015-i n . D\end{array}$ & 1.9134 & & & & & & \\
\hline $\mathrm{Ti}(12)$ & 69926 & & $\begin{array}{l}\text { Wire } \\
0.1 \text {-in. } L \times 0.020 \text {-in. } D\end{array}$ & 2.166 & & & & & & \\
\hline $0.1 \% \mathrm{AgAl}$ (13) & 03783 & Rx Wire & Metal Wire & 1.2550 & $v$ & 16.474 & 0.035 & 0.210 & 1 & \\
\hline $0.1 \%$ AuAT (14) & & Rx Wire & Metal Wire & 1.1948 & $v$ & 14.680 & 0.035 & 0.180 & $k$ & \\
\hline $\mathrm{MnO}_{2} \quad(15)$ & 55086 & & $\begin{array}{l}\text { Oxide Wire } \\
0.125-\text { in. } L \times 0.020-\text { in. D }\end{array}$ & 1.5017 & v & 17.968 & 0.035 & 0.240 & $T$ & \\
\hline $0.1 \% \operatorname{TaAl}(16)$ & 03783 & $217 \mathrm{~W}$ & $\begin{array}{l}\text { Metal Wire } \\
0.100-\text { in. } L \times 0.020-\text { in. D }\end{array}$ & 0.7340 & & & & & & \\
\hline $1.0 \% \operatorname{TaU}(16)$ & 32548 & ORNL & $0.100-$ in. L $\times 0.020$-in. $D$ & 2.899 & & & & & & \\
\hline SS (17) & & & 0.1 -in. $L \times 0.033$-in. $D$ & 10.307 & & & & & & EOTA SS Rod \\
\hline $\mathrm{Cu}(18)$ & 19047 & & $\begin{array}{l}\text { Metal Wire } \\
0.100-\text { in. } L \times 0.020-\text { in. D }\end{array}$ & 4.112 & & & & & & \\
\hline & & & & & & & & & & \\
\hline
\end{tabular}


TABLE $A-8$

RUN 97, CONTAINER F-7, SPECTRAL SET DETAILS

Test: Run 97

Page 1 of 2

HEDL Capsule Number

Container Identification $\mathrm{F}-7$

Set Identification:

$2:+\underline{59.44 \mathrm{~cm}}$

\begin{tabular}{|c|c|c|c|c|c|c|c|c|c|c|}
\hline \multirow[b]{2}{*}{ Material } & \multirow[b]{2}{*}{$\begin{array}{c}\text { Purchase Order } \\
\text { Number }\end{array}$} & \multirow[b]{2}{*}{$\begin{array}{l}\text { Batch } \\
\text { Nulnber }\end{array}$} & \multirow[b]{2}{*}{ Description } & \multirow{2}{*}{$\begin{array}{c}\text { Material } \\
\text { We ight } \\
\text { (mg) }\end{array}$} & \multicolumn{5}{|c|}{ Encassulated Monitor Description } & \multirow[b]{2}{*}{ Remarks } \\
\hline & & & & & $\ln$ & $\begin{array}{c}\text { Weight } \\
\text { (mo) }\end{array}$ & $\begin{array}{l}\text { Diam } \\
(\text { in. }) \\
\end{array}$ & $\begin{array}{l}\text { Lerigen } \\
\text { (in.) } \\
\end{array}$ & Ident & \\
\hline $1.0 \% \operatorname{CoV}(1)^{*}$ & Fabry & & $\begin{array}{l}\text { Metal wire } \\
0.1-\text { in. } L \times 0.015-\text { in. } D\end{array}$ & 1.9979 & & & & & & \\
\hline $\mathrm{Fe}(1)$ & 19046 & & $\begin{array}{l}\text { Metal Wire } \\
0.100-i n . L \times 0.020-i n . D\end{array}$ & 3.869 & & & & & & \\
\hline $\mathrm{Ni}(1)$ & 1 & & $\begin{array}{l}\text { Metal Wire } \\
0.1 \text {-in. } L \times 0.020-i n .0\end{array}$ & 4.299 & & & & & & \\
\hline $\mathrm{Cu}(1)$ & 19047 & & $\begin{array}{l}\text { Metal Wire } \\
0.1-\text { in. L } \quad 0.020-\text { in. D }\end{array}$ & 4.126 & & & & & & \\
\hline Ti (1) & 69926 & & $\begin{array}{l}\text { Metal Wire } \\
0.1-i n . L \times 0.020-i n .0\end{array}$ & 2.127 & & & & & & \\
\hline $\mathrm{Fe}(2)$ & 19046 & & $\begin{array}{l}\text { Metal Wire } \\
0.1 \text {-in. L } \times 0.020 \text {-in. D }\end{array}$ & 3.637 & & & & & & \\
\hline $\mathrm{Ni}(3)$ & 1 & & $\begin{array}{l}\text { Metal Wire } \\
0.1-i n . L \times 0.020-i n . D\end{array}$ & 4.340 & & & & & & \\
\hline $235 \mathrm{U}(4)$ & 55047 & $264-C$ & Dxide wire & 1.329 & V & 20.655 & 0.050 & 0.125 & 58 & Series 3 \\
\hline${ }^{237} \mathrm{~Np}(5)$ & 39407 & $\mathrm{~Np}-24-\mathrm{Hp}$ & pxide Wire & 6.451 & v & 51.771 & 0.050 & 0.345 & $24::$ & Series I \\
\hline${ }^{339} \mathrm{Pu}(6)$ & 39407 & 453-B0 & pxide wire & 1.467 & t & 44.869 & 0.050 & 0.280 & $26 \because$ & \\
\hline $\mathrm{Sc}_{2} \mathrm{O}_{3} \quad(7)$ & 39407 & DRNL-1 & pxide Wire & 1.364 & $v$ & 40.564 & 0.050 & 0.255 & 93 & \\
\hline $\begin{array}{l}0.1 \% \mathrm{Sc}_{2} \mathrm{O}_{3} / \mathrm{MgO} \\
(8)\end{array}$ & 55046 & 1 & $\begin{array}{l}\text { pxide Wire } \\
0.125 \text {-in. } L \times 0.020 \text {-in. } 0\end{array}$ & 0.9017 & y & 21.67 & 0.035 & 0.300 & $F$ & \\
\hline${ }^{10} \mathrm{~B} \quad(9)$ & 44051 & AI & Crystal & 0.980 & $y$ & 41.167 & 0.050 & 0.255 & $B 252-E D$ & \\
\hline 6 LiF (10) & 44051 & AI & Crystal & 2.484 & $N$ & 41.960 & 0.050 & 0.255 & $L 325-\times 5$ & \\
\hline
\end{tabular}

* Number in parentheses $=$ hole number in container. 


\section{TABLE A-8 (Continued)}

Test: Run 97

HEDL Capsule Number

Container Identification

F-7

\begin{tabular}{|c|c|c|c|c|c|c|c|c|c|c|}
\hline \multirow[b]{2}{*}{ Material } & \multirow[b]{2}{*}{$\begin{array}{c}\text { Purchase Order } \\
\text { Number }\end{array}$} & \multirow[b]{2}{*}{$\begin{array}{l}\text { Batch } \\
\text { Number }\end{array}$} & \multirow[b]{2}{*}{ Description } & \multirow{2}{*}{$\begin{array}{c}\text { Material } \\
\text { Weight } \\
\text { (mg) }\end{array}$} & \multicolumn{5}{|c|}{ Encapsulated Monitor Description } & \multirow[b]{2}{*}{ Remarks } \\
\hline & & & & & In & $\begin{array}{c}\text { Weight } \\
(\mathrm{mg})\end{array}$ & $\begin{array}{l}\text { Diam } \\
\text { (in.) }\end{array}$ & $\begin{array}{l}\text { Length } \\
\text { (in.: }\end{array}$ & Ident & \\
\hline $1.0 \% \operatorname{cov}(11)$ & Fabry & & $\begin{array}{l}\text { Thetal wire } \\
0.7 \text {-in. } \perp \times 0.015 \text {-in. } 0\end{array}$ & 1.9387 & & & & & & \\
\hline $\begin{array}{l}0.1 \% \text { CoMg0 } \\
\text { (ii) }\end{array}$ & 27940 & ORNL-1 & $\begin{array}{l}\text { 0xide Wire } \\
0.100-\text { in. } L \times 0.020 \text {-in. } 0\end{array}$ & 1.181 & $v$ & 20.68 & 0.035 & 0.300 & 7 & Series 4 \\
\hline $0.1 \% \operatorname{COAI}(11)$ & & SRM-953 & $\begin{array}{l}\text { Metal Wire } \\
0.100-\text { in. } L \times 0.020-i n .0\end{array}$ & 1.2030 & & & & & & NBS Roll I \\
\hline $1.0 \% \operatorname{cov}(12)$ & Fabry & & $\begin{array}{l}\text { Metal Wire } \\
0 . \text { Fin. } L \times 0.015-i n . D\end{array}$ & 1.8080 & & & & & & \\
\hline $\mathrm{Ti}(12)$ & 69926 & & $\begin{array}{l}\text { Metal Wire } \\
0.1-i n . L \times 0.020 \text {-in. D }\end{array}$ & 2.164 & & & & & & \\
\hline $0.1 \%$ AgAl (13) & 03783 & Rx Wire & Metal Wire & 1.1889 & $v$ & 16.978 & 0.035 & 0.210 & 0 & \\
\hline $0.1 \%$ AuAl (14) & & Rx Wire & Metal Wire & 1.2760 & $v$ & 14.388 & 0.035 & 0.180 & v & \\
\hline $\mathrm{Mn}_{3} \mathrm{O}_{4} \quad(15)$ & 55086 & & $\begin{array}{l}\text { Oxide Wire } \\
0.125-\text { in. } L \times 0.020-i n .0\end{array}$ & 1.3433 & $v$ & 17.847 & 0.035 & 0.240 & N & \\
\hline $0.1 \% \operatorname{TaA1}(16)$ & 03783 & 2176 & $\begin{array}{l}\text { Meta Wire } \\
0.1-\text { in. L } \times 0.020 \text {-in. D }\end{array}$ & 0.7136 & & & & & & \\
\hline 1.0\% TaV (16) & 32548 & ORNL & $0.1-$ in. $L \times 0.020$-in. 0 & 2.788 & & & & & & \\
\hline SS (17) & & & $0.1-$ in. $L \times 0.033$-in. 0 & 10.264 & & & & & & FOTA SS Rod \\
\hline $\mathrm{Cu}(18)$ & 19047 & & $\begin{array}{l}\text { Meta Wire } \\
0.1-i n . L \times 0.020-i n . D\end{array}$ & 4.016 & & & & & & \\
\hline & & & & & & & & & & \\
\hline & & & & & & & & & & \\
\hline
\end{tabular}


TABLE $A-9$

RUN 97, GRADIENT CONTAINERS 0 THROUGH 15, DOSIMETER DETAILS

Test: Run 97

Page 1 of 3

HEDL Capsule Number

Container Identification

Set Identification: Gradient Set Loading

$\mathrm{R}$

2:

\begin{tabular}{|c|c|c|c|c|c|c|c|c|c|c|}
\hline \multirow{3}{*}{$\begin{array}{l}\text { Material } \\
0.1 \% \operatorname{co} / \mathrm{MgO} \\
(0)^{*}\end{array}$} & \multirow{2}{*}{$\begin{array}{c}\text { Purchase Order } \\
\text { Number }\end{array}$} & \multirow[b]{2}{*}{$\begin{array}{l}\text { Batch } \\
\text { Number }\end{array}$} & \multirow[b]{2}{*}{ Description } & \multirow{2}{*}{$\begin{array}{c}\text { Material } \\
\text { Height } \\
\text { (mq) }\end{array}$} & \multicolumn{5}{|c|}{ Encapsulated Monitor Description } & \multirow[b]{2}{*}{ Reilarks } \\
\hline & & & & & In & $\begin{array}{l}\text { Weight } \\
\text { (mig) }\end{array}$ & $\begin{array}{l}\text { Diam } \\
\text { (in.) }\end{array}$ & $\begin{array}{l}\text { Length } \\
\text { (in.) }\end{array}$ & Ident & \\
\hline & 27940 & ORNL Tube 1 & $\begin{array}{l}\text { oxide Wire } \\
0.100-\text { in. } L \times 0.020-\text { in. } D\end{array}$ & 1.415 & $v$ & 19.36 & 0.035 & 0.300 & $F$ & Series 3 \\
\hline $\mathrm{Fe}(0)$ & 19046 & onim ture & $\begin{array}{l}\text { Metalire } \\
0.100-i n . L \times 0.020-i n . D\end{array}$ & 3.896 & & & & & & Rx Wire \\
\hline $\begin{array}{l}0.1 \% \mathrm{Co} / \mathrm{MgO} \\
\text { (i) }\end{array}$ & 27940 & ORNL Tube 1 & $\begin{array}{l}\text { Oxide Wire } \\
0.100-i n . L \times 0.020-i n .0\end{array}$ & 1.247 & v & 20.15 & 0.035 & 0.300 & 0 & Series 4 \\
\hline $\mathrm{Fe}(1)$ & 19046 & & $\begin{array}{l}\text { Metal Wire } \\
0.100-\text { in. } L \times 0.020-\text { in. } D\end{array}$ & 3.840 & & & & & & Rx Wire \\
\hline $\begin{array}{l}0.1 \% \text { Co } / \mathrm{MgO} \\
\text { (2) }\end{array}$ & 27940 & ORNL Tube 1 & $\begin{array}{l}\text { 0xide Wire } \\
0.100-i n . L \times 0.020-i n .0\end{array}$ & 1.114 & v & 20.32 & 0.035 & 0.300 & 8 & Series 4 \\
\hline $\mathrm{Fe}(2)$ & 19046 & & $\begin{array}{l}\text { Metal Wire } \\
0.100-\text { in. } L \times 0.020-\text { in. } D\end{array}$ & 3.762 & & & & & & Rx Wire \\
\hline $\begin{array}{l}0.1 \% \mathrm{Co} / \mathrm{MgO} \\
(3)^{2}\end{array}$ & 27940 & ORNL Tube 1 & $\begin{array}{l}\text { 0xide Wire } \\
0.100 \text {-in. } L \times 0.020 \text {-in. } 0\end{array}$ & 1.119 & v & 21.30 & 0.035 & 0.300 & v & Series 4 \\
\hline $\mathrm{Fe}(3)$ & 19046 & & $\begin{array}{l}\text { Metal Wire } \\
0.100-i n . L \times 0.020-i n .0\end{array}$ & 3.743 & & & & & & Rx Wire \\
\hline $\begin{array}{l}0.1 \% \mathrm{Co} / \mathrm{MgO} \\
(4) \text {. }\end{array}$ & 27940 & ORNL Tube 1 & $\begin{array}{l}\text { oxide Wire } \\
0.100-\text { in. } L \times 0.020-i n .0\end{array}$ & 1.222 & v & 20.67 & 0.035 & 0.300 & 2 & Series 4 \\
\hline $\mathrm{Fe}(4)$ & 19046 & & $\begin{array}{l}\text { Metal Wire } \\
0.100-\text { in. } L \times 0.020-\text { in. } D\end{array}$ & 3.794 & & & & & & Rx Wire \\
\hline $\begin{array}{l}0.1 \% \operatorname{Co} / \mathrm{Mg} 0 \\
(5)\end{array}$ & 27940 & ORNL Tube 1 & $\begin{array}{l}\text { Oxide Wire } \\
0.100-\text { in. } L \times 0.020-\text { in. } D\end{array}$ & 1.112 & $v$ & 20.95 & 0.035 & 0.300 & $T$ & Series 4 \\
\hline $\mathrm{Fe}(5)$ & 19046 & & $\begin{array}{l}\text { Metal Wire } \\
0.100-\text { in. } L \times 0.020 \text {-in. D }\end{array}$ & 3.836 & & & & & & Rx wire \\
\hline $\begin{array}{l}0.1 \% \mathrm{Co} / \mathrm{Mg} 0 \\
(6)^{2}\end{array}$ & 27940 & ORNL Tube 1 & $\begin{array}{l}\text { 0xide Wire } \\
0.100 \text {-in. } L \times 0.020 \text {-in. } 0\end{array}$ & 1.192 & $v$ & 21.84 & 0.035 & 0.300 & $s$ & Series 4 \\
\hline $\mathrm{Fe}(6)$ & 19046 & & $\begin{array}{l}\text { Metal Wire } \\
0.100-\text { in. } L \times 0.020-i n .0\end{array}$ & 3.691 & & & & & & Rx wire \\
\hline
\end{tabular}

*Number in parentheses $=$ Set Number. 


\section{TABLE A-9 (Continued)}

Test: Run 97

HEOL Capsule Number

Container Identification

Set Identification: Gradient Set Loading

Page 2 of 3

\begin{tabular}{|c|c|c|c|c|c|c|c|c|c|c|}
\hline \multicolumn{11}{|c|}{ Container Identification } \\
\hline \multirow[b]{2}{*}{ Material } & \multirow[b]{2}{*}{$\begin{array}{c}\text { Purchase Order } \\
\text { Number }\end{array}$} & \multirow[b]{2}{*}{$\begin{array}{l}\text { Batch } \\
\text { Number }\end{array}$} & \multirow[b]{2}{*}{ Description } & \multirow{2}{*}{$\begin{array}{l}\text { Material } \\
\text { Height } \\
\text { (mq) }\end{array}$} & \multicolumn{5}{|c|}{ Encapsulated Monitor Description } & \multirow[b]{2}{*}{ Remarks } \\
\hline & & & & & In & $\begin{array}{c}\text { Weight } \\
\text { (ng) }\end{array}$ & $\begin{array}{l}\text { Diam } \\
\text { (in.) }\end{array}$ & $\begin{array}{l}\text { Length } \\
\text { (in.) }\end{array}$ & Ident & \\
\hline $\begin{array}{l}0.7 \% \text { Co/MgO } \\
\text { (i) }\end{array}$ & 27940 & ORNL Tube 1 & $\begin{array}{l}\text { Oxide Wire } \\
0.100 \text {-in. } 1 \times 0.020-\text { in. } D\end{array}$ & 1.134 & v & 21.06 & 0.035 & 0.300 & 3 & Series 4 \\
\hline $\mathrm{Fe} \mathrm{(7)}$ & 19046 & & $\begin{array}{l}\text { Metal Wire } \\
0.100-\text { in. } L \times 0.020-i n . D\end{array}$ & 4.005 & & & & & & Rx Wire \\
\hline$(8)^{0.1 \% \mathrm{Co} / \mathrm{MgO}}$ & 27940 & ORNL Tube 1 & $\begin{array}{l}\text { Oxide Wire } \\
0.100-\text { in. } L \times 0.020-i n .0\end{array}$ & 1.189 & v & 20.16 & 0.035 & 0.300 & $\mathrm{~J}$ & Series 4 \\
\hline $\mathrm{Fe}(8)$ & 19046 & & $\begin{array}{l}\text { Metal Wire } \\
0.100-\text { in. } L \times 0.020-i n . D\end{array}$ & 4.079 & & & & & & Rx Wire \\
\hline$(9)$ Co/MgO & 27940 & ORNL Tube 1 & $\begin{array}{l}\text { Oxide Wire } \\
0.100-\text { in. } L \times 0.020-i n . D\end{array}$ & 0.994 & 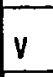 & 19.28 & 0.035 & 0.300 & $N$ & Series 4 \\
\hline Fe (9) & 19046 & & $\begin{array}{l}\text { Metal Wire } \\
0.100-\text { in. } L \times 0.020-i n .0\end{array}$ & 3.948 & & & & & & Rx Wire \\
\hline $\begin{array}{l}0.1 \% \mathrm{Co} / \mathrm{MgO} \\
(10)\end{array}$ & 27940 & ORNL Tube 1 & $\begin{array}{l}\text { Oxide Wire } \\
0.100-\text { in. } L \times 0.020-i n .0\end{array}$ & 0.954 & $v$ & 20.00 & 0.035 & 0.300 & $K$ & Series 4 \\
\hline $\mathrm{Fe}(10)$ & 19046 & & $\begin{array}{l}\text { Metal Wire } \\
0.100-i n . L \times 0.020-i n . D\end{array}$ & 3.696 & & & & & & Rx Wire \\
\hline $\begin{array}{l}0.1 \% \text { Co/MgO } \\
\text { (i1) }\end{array}$ & 27940 & ORNL Tube 1 & $\begin{array}{l}\text { Oxide Wire } \\
0.100-\text { in. } L \times 0.020-i n . D\end{array}$ & 1.053 & v & 18.62 & 0.035 & 0.300 & 6 & Series 4 \\
\hline $\mathrm{Fe}(11)$ & 19046 & & $\begin{array}{l}\text { Metal Wire } \\
0.100-\text { in. } L \times 0.020-i n . D\end{array}$ & 4.049 & & & & & & Rx Wire \\
\hline $\begin{array}{l}0.1 \% \text { Co/MgO } \\
(12)\end{array}$ & 27940 & ORNL Tube 1 & $\begin{array}{l}\text { 0xide Wire } \\
0.100 \text {-in. L } \times 0.020 \text {-in. } 0\end{array}$ & 1.486 & $v$ & 20.21 & 0.035 & 0.300 & 4 & Series 4 \\
\hline $\mathrm{Fe} \mathrm{(12)}$ & 19046 & & $\begin{array}{l}\text { Metal Wire } \\
0.100-\text { in. } L \times 0.020-\text { in. } D\end{array}$ & 3.615 & & & & & & Rx Wire \\
\hline $\begin{array}{l}0.1 \% \text { Co } \mathrm{MgO} \\
(13)\end{array}$ & 27940 & ORNL Tube 1 & $\begin{array}{l}\text { Oxide Wire } \\
0.100 \text {-in. } L \times 0.020 \text {-in. } D\end{array}$ & 1.071 & $v$ & 18.89 & 0.035 & 0.300 & 2 & Series 4 \\
\hline $\mathrm{Fe}(13)$ & 19046 & & $\begin{array}{l}\text { Metal Wire } \\
0.100-\text { in. } L \times 0.020-i n . D\end{array}$ & 3.944 & & & & & & Rx Wire \\
\hline
\end{tabular}


TABLE A-9 (Continued)

Test: Run 97

HEOL Capsule Number

Container Identification

Set Identification: Gradient Set Loading

Page 3 of 3

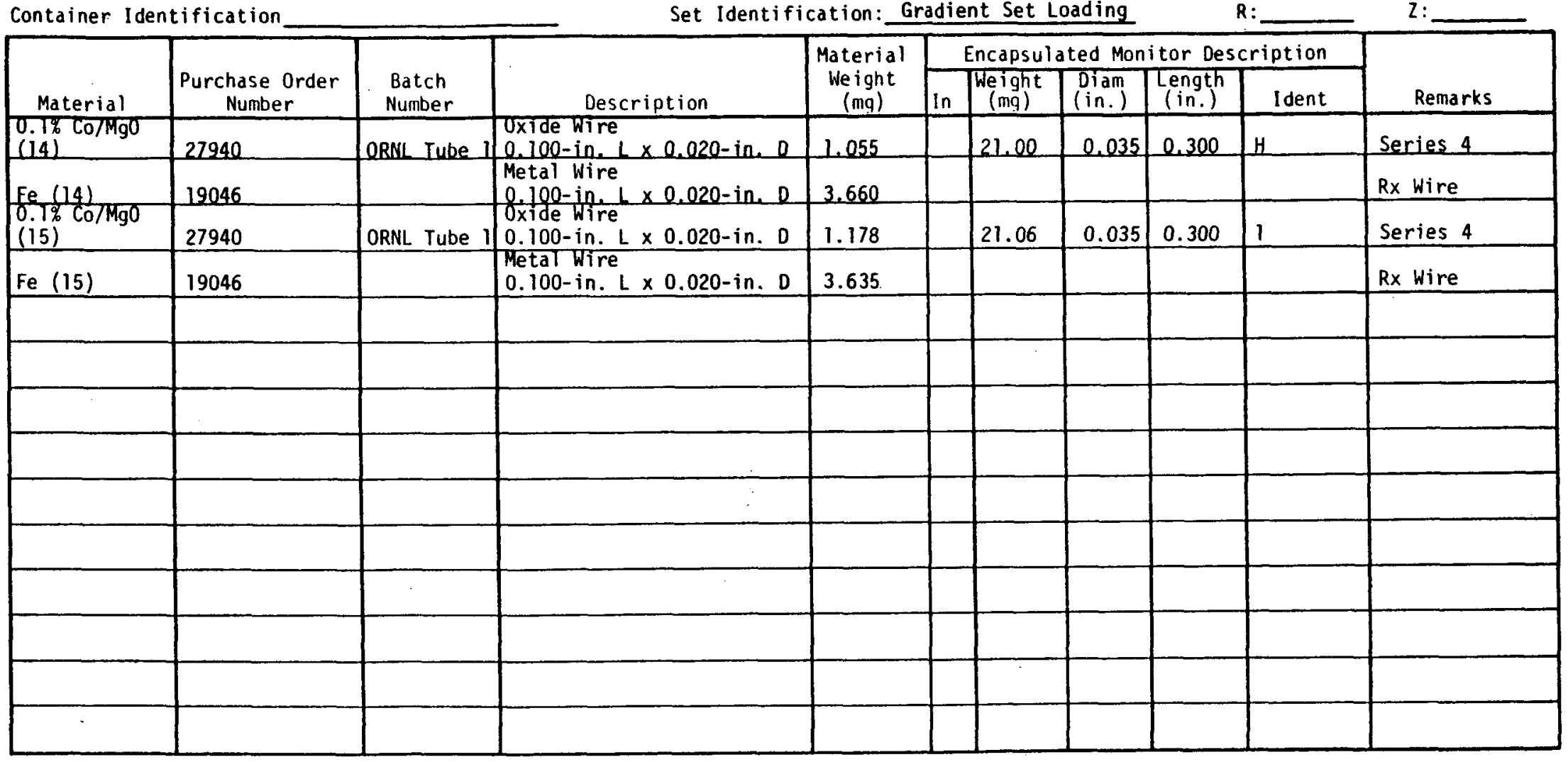




\section{APPENDIX B}

RUN 97 HELIUM ACCUMULATION FLUENCE MONITORS SPECIFIC DETAILS 


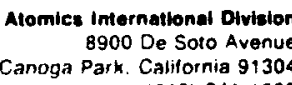

June 26, 1978

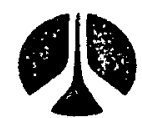

Rockwell International

Mr. J. A. Ul seth

Building 325,300 Area

Hanford Engineering Development Laboratory

Westinghouse Hanford Company

Post Office Box 1970

Richland, Washington 99352

Dear Jim:

Subject: Nine Capsules for Irradiation in the EBR-II

Run 97 Dosimetry Experiment

[nclosed are nine stainless steel capsules to be irradiated at three Row 2 axial locations during the EBR-II Run 97 Dosimetry Experiment. The irradiation capsules contain a variety of helium accumulation fluence monitors (HAFM's), made of vanadium, niobium, platinum, and Type 304 stainless steel, along with a number of pure element wires and metal alloys.

\section{Purposes of Tests}

The purposes of the experiment are threefold: 1) to demonstrate sets of advanced energy-dependent HAF!'s in Row 2 locations, for fluences several times greater than those used before; 2) to determine the effectiveness of vanadium and the alternative materials $\mathrm{Nb}, \mathrm{Pt}$, and $\mathrm{SS}$ as HAFM encapsulating materials for containing generated helium; and 3 ) to determine whether HAFM's can be irradiated in an atmosphere of helium (for thermal coupling) without this helium affecting the results.

\section{Selection of Reactor Locations}

The desire to have several different neutron energy distributions has led to the selection of three locations, each adjacent to a HEDL fluxspectral multiple foil capsule. Emphasis was placed on having the capsules away from any vertical reactor discontinuity so that the fluxspectrum could be determined as well as possible and with minimum gradients. Requested locations in Row 2 are as follows:

\begin{tabular}{|c|c|c|c|}
\hline Capsule Group & Capsules & Z-Location $(\mathrm{cm})$ & Desired Conditions \\
\hline Al $-1+3$ & $B, P, O$ & $+39.4 \mathrm{~cm}$ & Maximum Temperature \\
\hline AI -2 & $A, H, K$ & $+1.9 \mathrm{~cm}$ & Maximum Flux \\
\hline$A I-\not z l \mid$ & $C, E, N$ & $-34.7 \mathrm{~cm}$ & Minimum Temperature \\
\hline
\end{tabular}


78AT -5880

June 26, 1978

Page 2

A summary of the masses of all materials in each of the nine capsules is provided in Table 1.

\section{Capsule Description}

The three capsule groups each contain three capsules. Each group includes an argon-filled 0.44-in. 00 capsule, 0.89-in. long, and two 0.128-in. 00 capsules, $0.88-i n$. Iong with holes to allow the plenum helium to penetrate. The dimensions of the two capsule types are given in the enclosed Figure 1 and are designed to fit within one of your "Specimen Holder, Parts 4 or 5" (HEDL Drawing H-3-44071, April 3, 1978).

We have loaded the nine capsules with a variety of materials described in Sections 4 and 5 of this letter. The three large capsules have been evacuated, back-filled with argon, and TIG-welded under argon. After welding, the capsules were cleaned, heated under vacuum to $500^{\circ} \mathrm{C}$ for one hour, and leak checked using the boiling water method. The smaller capsules have press-fitted ends, and a hole in the side to allow the helium within the pin to penetrate and bathe the contents during the irradiation. Each capsule has been engraved with an identification letter (on top of the large capsules, on the side of the small capsules) and an arrow which should point toward the zenith while in EBR-II. At HEOL, the capsules are to be inserted into the three HEDL subcapsules, along with HEDL flux gradient wires.

\section{Contents of the Large Capsules}

The three large capsules have been loaded with materials to study Items 1 and 2 of Section 1. In order to determine the effectiveness of various encapsulating materials for containing generated helium, the large capsules contain a variety of HAFM's with the capsule materials made of vanadium, niobium, platinum, and Type 304 stainiess steel. Boron is included in the HAFM's to generate quantities of helium and build up a helium pressure within the HAFM capsules. Many of these four types of HAFM's (having $0.035-i n .00)$ are also further encapsulated in 0.050-in. 00 capsules made of Pt and $V$. Subsequent shearing of the outer capsules is expected to provide information on possible permeation or leakage of helium through the inner capsule walls. Subsequent helium analyses of the inner capsules might include experiments to heat the HAFM's in steps, or etching experiments to determine the depth of helium penetration into the inner walls of the different capsule materials.

Several special vanadium capsules, also containing boron at one end, also contain 0.020 in. wires of $\mathrm{V}, \mathrm{Nb}, \mathrm{Pt}$, and stainless steel. Each capsule has a variable (stepped) inside diameter, which provides a different depth of helium surrounding the wire. Measurement of the 
78AT -5880

June 26,1978

Page 3

helium at various locations along the wire lengths will show whether the helium is being simply absorbed, or is being knocked into the wire by neutron collisions with the surrounding helium gas.

The other principal experiment included in the midplane large capsule (Capsule $A$ ) is the demonstration of a set of advanced energy-dependent HAFM's for fluence levels several times greater than those obtained during the Run 75D dosimetry test. HAFM capsules, made with vanadium, niobium and platinum have been filled with the most promising energysensitive heljum generation materials. Capsule $A$ contains at least one each of $L i F, 6_{L i F}, 7_{L i F}, \mathrm{Be}, \mathrm{B}, \mathrm{C}, \mathrm{NaF}, \mathrm{NaCl}, \mathrm{Al}, \mathrm{Si}, \mathrm{SiO}_{2}, \mathrm{KCl}, \mathrm{Kl}, \mathrm{CaF}$, $\mathrm{Ti}, \mathrm{TiN}, \mathrm{V}, \mathrm{V}_{2} \mathrm{O}_{5}, \mathrm{Fe}, \mathrm{FeF}_{2}, \mathrm{Ni}, \mathrm{Cu}, \mathrm{Zr}, \mathrm{ZrN}, \mathrm{Nb}, \mathrm{Pt}$, and PbS. These HAFM's have been sealed by EB-welding, leak checked, and generally fabricated according to the "Specifications for Helium Accumulation Fluence Monitors" by H. Farrar IV, January 10, 1977. Molybdenum, and its seven enriched isotopes, and iron and the enriched isotopes $54 \mathrm{Fe}$, $56 \mathrm{Fe}$ and $57 \mathrm{Fe}$ are also included in Capsule $\mathrm{A}$ in an unsealed inner stainless steel tube.

The results of the helium analyses of these HAFM's will be correlated with ENDF/B cross section data, and compared with the results of HEDL radionetric foil reactions that have equivalent energy responses.

\section{Contents of the Sinall Capsules}

The six snall capsules are designed to study Item 3) of Section 1. The capsules are not sealed, and this will therefore allow the helium from the pin to enter while they are in the reactor. Since the pin will be loaded at STP, the helium pressure at reactor temperature will be -3 atmospheres.

All the small capsules were loaded with bare wires of $\mathrm{V}, \mathrm{Nb}, \mathrm{Pt}$, and stainless steel, having dimensions between 0.020 - and 0.050 -inches. Solne unsealed "stepped" vanadium capsules containing wires were al so included. After the irradiation, the wires will undergo various etching procedures to determine the depth of helium penetration, if any. If this penetration is small, it will be feasible to irradiate HAFM's made of that material in a helium atmosphere, which provides a suitable thermal coupling. If there is helium penetration after such an irradiation, the HAFM capsules would be etched to remove the surface material contaminated with helium, so this source of helium would not affect the resuits. 
TBAT -5880

June 26,1978

Page 4

This irradiation is a very important part of our plans to develop and demonstrate helium accumulation fluence monitors, and we are particularly pleased to be able to include our capsules at locations close to HEDL's major dosimetry sets.

Yours truly,

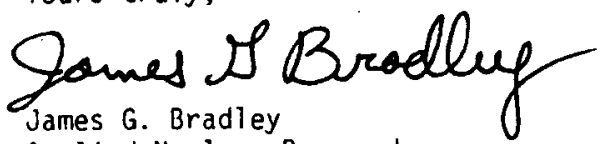

Applied Nuclear Research

Energy Systems Group

$737-E .56 / p a g$

Enclosures

CC w/enclosures:

J. W. Lewellen, DOE-RRT-HQ

E. P. Lippincott, HEDL

W. N. MCEIroy, HEDL 
TABLE 7

Enclosure 1 to AI Letter 78AT-5880

SUMMARY OF ROCKWELL INTERNATIONAL MATERIALS FOR RUN 97 June 26, 1978

\begin{tabular}{|c|c|c|c|c|c|c|c|c|c|c|}
\hline \multirow{3}{*}{$\begin{array}{l}\text { Element } \\
\text { or } \\
\text { Isotope }\end{array}$} & \multirow[b]{3}{*}{ Chemical or Physical Form } & \multicolumn{9}{|c|}{ Mass of Element or Isotope } \\
\hline & & \multicolumn{3}{|c|}{$\begin{array}{l}\text { Group AI-2 } \\
\text { (Midplane) }\end{array}$} & \multicolumn{3}{|c|}{$\begin{array}{l}\text { Group AI - } \\
(+39.4 \mathrm{~cm})\end{array}$} & \multicolumn{3}{|c|}{$\begin{array}{l}\text { Group AI-3 } \\
(-37.4 \mathrm{~cm})\end{array}$} \\
\hline & & $A$ & $\mathrm{H}$ & $K$ & $B$ & & 0 & & & $N$ \\
\hline${ }^{6} \mathrm{Li}$ & ${ }^{6} \mathrm{LiF}, \mathrm{N}_{\mathrm{LiF}}$ & 0.3 & & & & & & & & \\
\hline $\begin{array}{l}\mathrm{C} L i \\
\mathrm{Be} \\
\mathrm{B} \\
\mathrm{C}\end{array}$ & $\begin{array}{l}\text { 'Lif, Nif } \\
\text { Chunks } \\
\text { Boron Crystals, Ni wire } \\
\text { Diamond }\end{array}$ & $\begin{array}{l}1.9 \\
6.2 \\
1.9 \\
1.5\end{array}$ & & & 1.2 & & & 1.0 & & \\
\hline $\begin{array}{l}N \\
0 \\
\mathrm{~F} \\
\mathrm{Na} \\
\mathrm{Al}\end{array}$ & $\begin{array}{l}\mathrm{TiN}, \mathrm{ZrN} \\
\mathrm{SiO}_{2}, \mathrm{~V}_{2} \mathrm{O}_{5} \\
\mathrm{LiF}, \mathrm{NaF}_{2} \mathrm{CaF}_{2}, \mathrm{FeF}_{2} \\
\mathrm{NaF}, \mathrm{NaCi} \\
\text { Wire }\end{array}$ & $\begin{array}{r}10.2 \\
7.2 \\
16.5 \\
6.8 \\
6.0\end{array}$ & & & & & & & & \\
\hline $\begin{array}{l}\mathrm{Si} \\
\mathrm{S} \\
\mathrm{Cl} \\
\mathrm{K} \\
\mathrm{Ca}\end{array}$ & $\begin{array}{l}\mathrm{Si}, \mathrm{SiO}_{2} \\
\mathrm{PbS} \\
\mathrm{NaCl}, \mathrm{KCl} \\
\mathrm{KCl}, \mathrm{KI} \\
\mathrm{CaF}_{2}\end{array}$ & $\begin{array}{l}7.3 \\
2.0 \\
5.7 \\
3.5 \\
3.4\end{array}$ & & & & & & & & \\
\hline $\begin{array}{l}\mathrm{Ti} \\
\mathrm{v} \\
\mathrm{Fe} \\
\mathrm{Ni} \\
\mathrm{Cu}\end{array}$ & $\begin{array}{l}\text { Wire, Tin } \\
\text { Wire, Capsules, } \mathrm{V}_{2} \mathrm{O}_{5} \\
\text { Wire, Chunks } \mathrm{FeF}_{2} \\
\text { Wire } \\
\text { Wire }\end{array}$ & $\begin{array}{r}13.4 \\
1724.1 \\
47.0 \\
16.2 \\
17.2\end{array}$ & 190.6 & 126.1 & $\begin{array}{r}442.7 \\
44.7\end{array}$ & 166.7 & 77.2 & 470.9 & 188.1 & 76.7 \\
\hline $\begin{array}{l}\mathrm{Zr} \\
\mathrm{Nb} \\
\mathrm{Mo} \\
\mathrm{I} \\
\mathrm{Pt}\end{array}$ & $\begin{array}{l}\text { Wire, ZrN } \\
\text { Wire, Capsules } \\
\text { Chunks } \\
\text { KI } \\
\text { Wire, Capsules }\end{array}$ & $\begin{array}{r}73.5 \\
1590.7 \\
55.8 \\
4.8 \\
4868.3\end{array}$ & $\begin{array}{r}76.8 \\
112.2\end{array}$ & 167.8 & $\begin{array}{r}120.6 \\
1151.3\end{array}$ & $\begin{array}{r}41.7 \\
196.9\end{array}$ & $\begin{array}{l}137.7 \\
258.5\end{array}$ & $\begin{array}{r}138.3 \\
1127.4\end{array}$ & $\begin{array}{r}78.3 \\
111.8\end{array}$ & $\begin{array}{r}97.7 \\
178.8\end{array}$ \\
\hline $\begin{array}{l}\mathrm{Pb} \\
\mathrm{SS}\end{array}$ & $\begin{array}{l}\text { PbS } \\
\text { Wire, Capsules, Wool }\end{array}$ & $\begin{array}{r}12.9 \\
5793.3\end{array}$ & 558.1 & 566.40 & 5371.7 & 574.0 & 561.3 & 5380.7 & 535.9 & 604.3 \\
\hline
\end{tabular}

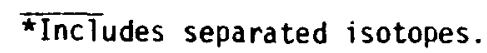


Enclosure 2 to

AI Letter 78AT-5880

June 26,1978

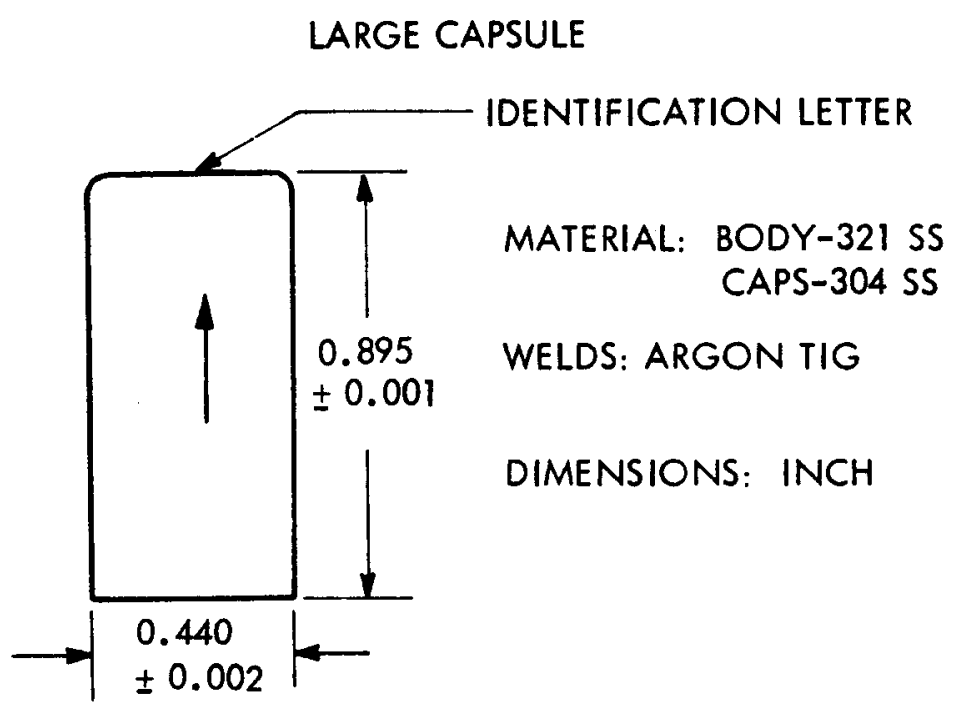

SMALL CAPSULE

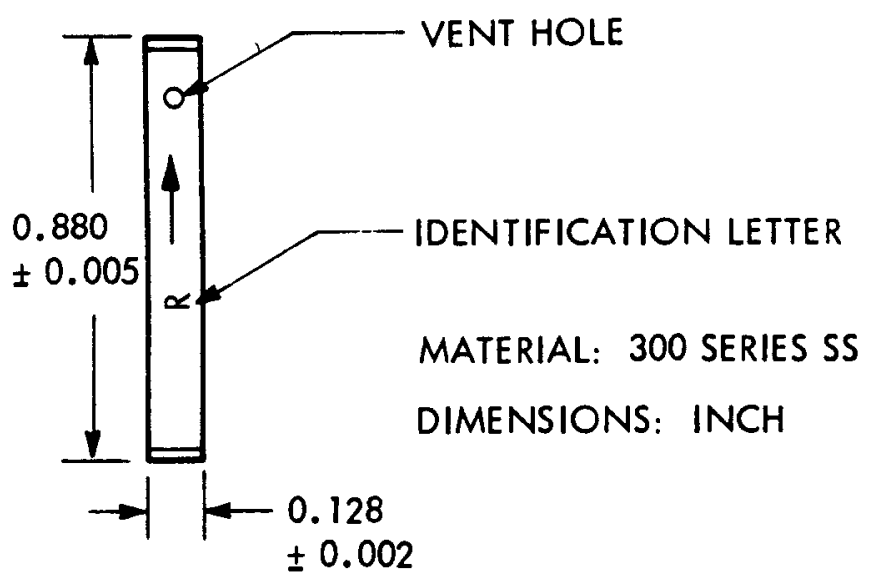

FIGURE B-1. Large and Small Capsules Containing Rockwell International Materials for EBR-II Run 97. 


\section{APPENDIX $C$}

RUN 97 SOLID STATE TRACK RECORDERS SPECIFIC DETAILS 


\section{RUN 97 SOLID STATE TRACK RECORDERS}

Three capsules containing solid state track recorders (SSTR) were included. Each capsule contained a total of six natural quartz crystal SSTR and two pieces of Marz-grade nickel. Quartz crystal SSTR faces were placed next to the nickel wafers to monitor the fissionable element content of the nickel. Quartz-quartz interfaces were also present so that the fissionable element content of the quartz itself could be measured.

Each set of six SSTR consisted of three pieces of natural quartz cut along the $001 \mathrm{plane}$ and three cut along the $100 \mathrm{plane}$. Three surfaces of each type of quartz were exposed to normally incident ${ }^{252} \mathrm{Cf}$ fission fragments, and three surfaces of each type were exposed to isotopically incident ${ }^{252} \mathrm{Cf}$ fission fragments to study the track relation characteristics of quartz crystal at the elevated temperature and high fluence of EBR-II.

The blank quartz surfaces will be exposed to ${ }^{252} \mathrm{Cf}$ fission fragments after the irradiation to study any possible changes in track recording properties of the SSTR. 
TABLE C-1

RUN 97, SSTR SET S-1 DETAILS

Test: Run 97 SSIR

HEDL Capsuie Number $\mathrm{S}-1$

Container Identification I

Set Identification:

\begin{tabular}{|c|c|c|c|c|c|c|c|c|c|c|}
\hline \multicolumn{10}{|c|}{ Container Iden } & \multirow{3}{*}{ 2: } \\
\hline \multirow[b]{2}{*}{ Material } & \multirow[b]{2}{*}{$\begin{array}{l}\text { Purchase Order } \\
\text { Number }\end{array}$} & \multirow[b]{2}{*}{$\begin{array}{l}\text { Batch } \\
\text { Number }\end{array}$} & \multirow[b]{2}{*}{ Description } & \multirow{2}{*}{$\begin{array}{c}\text { Material } \\
\text { Weight } \\
\text { (ma) }\end{array}$} & \multicolumn{5}{|c|}{ Encapsulated Monitor Description } & \\
\hline & & & & & In & 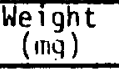 & $\begin{array}{l}\text { Diam } \\
\text { (in.) }\end{array}$ & $\begin{array}{l}\text { Lengtri } \\
\text { in. } i\end{array}$ & Ident & \\
\hline $\mathrm{Ni}$ & & MRC & Marz Grade & & & & & & $\mathrm{T}$ & \\
\hline Quartz & & VALTEC & & & & & & & -0 & \\
\hline Quartz & & VALTEC & & & & & & & -1 & \\
\hline Quartz & & VALTEC & & & & & & & -2 & \\
\hline Quartz & & VALTEC & & & & & & & -3 & \\
\hline Quartz & & VALTEC & & & & & & & -4 & \\
\hline Quartz & & VALTEC & & & & & & & -5 & \\
\hline Ni & & MRC & Marz Grade & & & & & & B & \\
\hline & & & & & & & & & & \\
\hline & & & & & & & & & & \\
\hline & & & & & & & & & & \\
\hline & & & & & & & & & & \\
\hline & & & & & & & & & & \\
\hline & & & & & & & & & & \\
\hline
\end{tabular}

$\stackrel{i}{\sim}$ 
TABLE C-2

RUN 97, SSTR SET S-2 DETAILS

Test: Run 97 SSTR

HEDL Capsule Number S-2

Container Identification 2

(2)

\begin{tabular}{|l|l|}
\hline Material & $\begin{array}{c}\text { Purchase Order } \\
\text { Number }\end{array}$ \\
\hline Ni & \\
\hline Quartz & \\
\hline
\end{tabular}

Quartz

Quartz

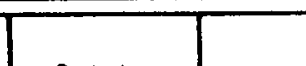

Set Identification

Page 1 of 1

\begin{tabular}{|l|l|}
\hline Quartz & \\
\hline Quartz & \\
\hline
\end{tabular}

Quart

Ni

\begin{tabular}{c|c} 
Batch \\
Number
\end{tabular} Description

Material
Height

\begin{tabular}{l|l} 
MRC & Marz Grade
\end{tabular}

VALTEC

VALTEC

VALTEC

VALTEC

VALTEC

VALTEC

\begin{tabular}{|l|l|l|}
\hline & & MRC \\
\hline & & \\
\hline & & \\
\hline & & \\
\hline
\end{tabular}

Marz Grade

(ma)

\begin{tabular}{|c|c|c|c|c|}
\hline \multicolumn{4}{|c|}{ Encapsulated Monitor Descr } \\
\hline In & $\begin{array}{c}\text { Weight } \\
\text { (mg) }\end{array}$ & $\begin{array}{c}\text { Diam } \\
\text { (in.) }\end{array}$ & $\begin{array}{c}\text { Length } \\
\text { (in.) }\end{array}$ & \\
\hline & & & & $T$
\end{tabular}

Z:

ription Ident Reinarks

\begin{tabular}{|l|l|l|l|l|l|l|l|l|l|l|l|}
\hline & & & & & & & & & & & \\
\hline
\end{tabular}


TABLE $\mathbf{C}-\mathbf{3}$

RUN 97, SSTR SET 3 DETAILS

Test: Run 97 SSTR

HEDL Capsule Number S-3

Container Identification 3

Page 1 of 1

\begin{tabular}{|c|c|c|c|c|c|c|c|c|c|c|}
\hline \multicolumn{10}{|c|}{ 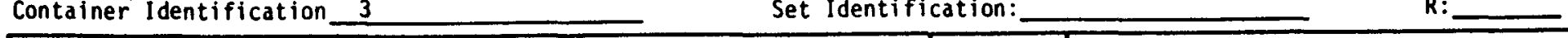 } & \multirow[b]{3}{*}{ Remarks } \\
\hline \multirow[b]{2}{*}{ Material } & \multirow[b]{2}{*}{$\begin{array}{c}\text { Purchase order } \\
\text { Number }\end{array}$} & \multirow[b]{2}{*}{$\begin{array}{l}\text { Batch } \\
\text { Number } \\
\end{array}$} & \multirow[b]{2}{*}{ Description } & \multirow{2}{*}{$\begin{array}{c}\text { Material } \\
\text { Weight } \\
\text { (mg) } \\
\end{array}$} & \multicolumn{5}{|c|}{ Encapsulated Monitor Description } & \\
\hline & & & & & In & $\begin{array}{l}\text { Weight } \\
\text { (ma) } \\
\end{array}$ & $\begin{array}{l}\text { Diam } \\
\text { (in.) }\end{array}$ & $\begin{array}{l}\text { Length } \\
\text { (in.) } \\
\end{array}$ & Ident & \\
\hline $\mathrm{Ni}$ & & MRC & Marz Grade & & & & & & $T$ & \\
\hline Quartz & & VALTEC & & & & & & & 0 & \\
\hline Quartz & & VALTEC & & & & & & & 1 & \\
\hline Quartz & & VALTEC & & & & & & & 2 & \\
\hline Quartz & & VALTEC & & & & & & & 3 & \\
\hline Quartz & & VAL TEC & & & & & & & 4 & \\
\hline Quartz & & VALTEC & & & & & & & 5 & \\
\hline $\mathrm{Ni}$ & & MRC & Marz Grade & & & & & & B & \\
\hline & & & & & & & & & & \\
\hline & & & & & & & & & & \\
\hline & & & & & & & & & & \\
\hline & & & & & & & & & & \\
\hline & & & & & & & & & & \\
\hline & & & & & & & & & & \\
\hline
\end{tabular}

$\stackrel{i}{i}$ 
APPENDIX D

EUROPEAN DOSIMETERS - SPECIFIC DETAILS 


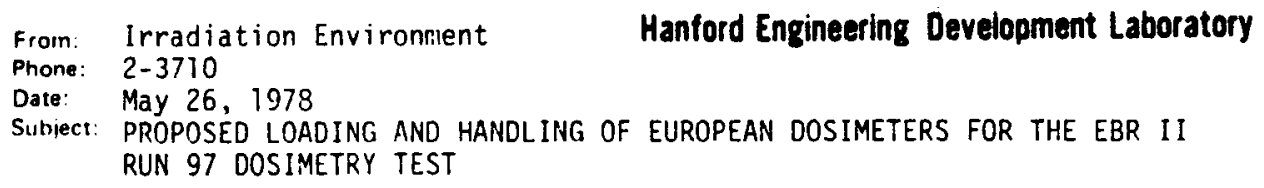

Subject: PROPOSED LOADING AND HANDLING OF EUROPEAN DOSIMETERS FOR THE EBR II RUN 97 DOSIMETRY TEST

To:
W. N. MCEl roy
CC: EP Lippincott
JAU/File/LB
Run $97 \mathrm{File/3}$
The Belgian, French, and German dosimeters will be loaded into the Run 97 Dosimetry Experiment Pin in the following manner:
Distance from
Reactor Mid-
plane, In.
Foils
Wires
Fission (1) Type of
$-23.4,15.5,23.4$
$\mathrm{Fe}, \mathrm{Ni}, \mathrm{Cu}, \mathrm{Ti}-\operatorname{Dos}^{(2)}$
$\mathrm{Nb}, 1 \% \mathrm{CO} / \mathrm{V}$
$-14.4,0$
$-$
$\mathrm{Fe}, \mathrm{Ni}, \mathrm{Cu}, \mathrm{Ti}$
$\mathrm{Nb}, 1 \% \mathrm{Co} / \mathrm{V}$
$238 u, 235 u$ Dos
$0,-15$.
$10 \mathrm{Nb}$ (MPI) ${ }^{(3)}$ and
$2{ }^{3} \mathrm{~Np}$
Dos
$0 .-15.4$

$$
\text { Ti Spacers }(5)
$$
$-$
SSTR 1 (4)
$0,-15.4$
2 Nb (France),
Ti Spacers, Cu
$-\operatorname{SSTR}^{(4)}$
$\mathrm{Ti}, \mathrm{Ni}, \mathrm{Fe}$
$0,-15.4$
$3 \mathrm{Nb}(\text { commercial })^{(6)}$..
$-$
SSTR $3^{(4)}$
$\int_{\text {3. A. Ulseth }}$

(1)-(6) See these footnotes on following page. 
(1) The fission monitors are being provided by HEDL, they are an oxide wire encapsulated in 0.050 -inch diameter Vanadium.

(2) Dos refers to a type of container used to hold dosimeter wires and capsules i.e. a right circular cylinder with several small holes drilled to contain the dosimeters. A common lid secured by a threaded screw holds the dosimeter in place.

(3) MPI - Max Planck Institute.

(4) SSTR - refers to the dosimeter container system for the foils - sec attached figure. The different types of $\mathrm{Nb}$ foils will be separated into SSTR 1,2, and 3 at each axial leve1. The foils will be stacked into the smaller containers and the positions recorded. After irradiation these small containers will be opened at $\mathrm{Mol}$ and the $\mathrm{Nb}$ destined for HEOL will be shipped to the US.

(5) Ti spacers - See drawing.

(6) Commercial - One Nb foil from three different companies i.e.: Highways Int., Goodfellow ilet., and Kawechi Chem. 
Nb FOIL SAMPLE CONTAINER

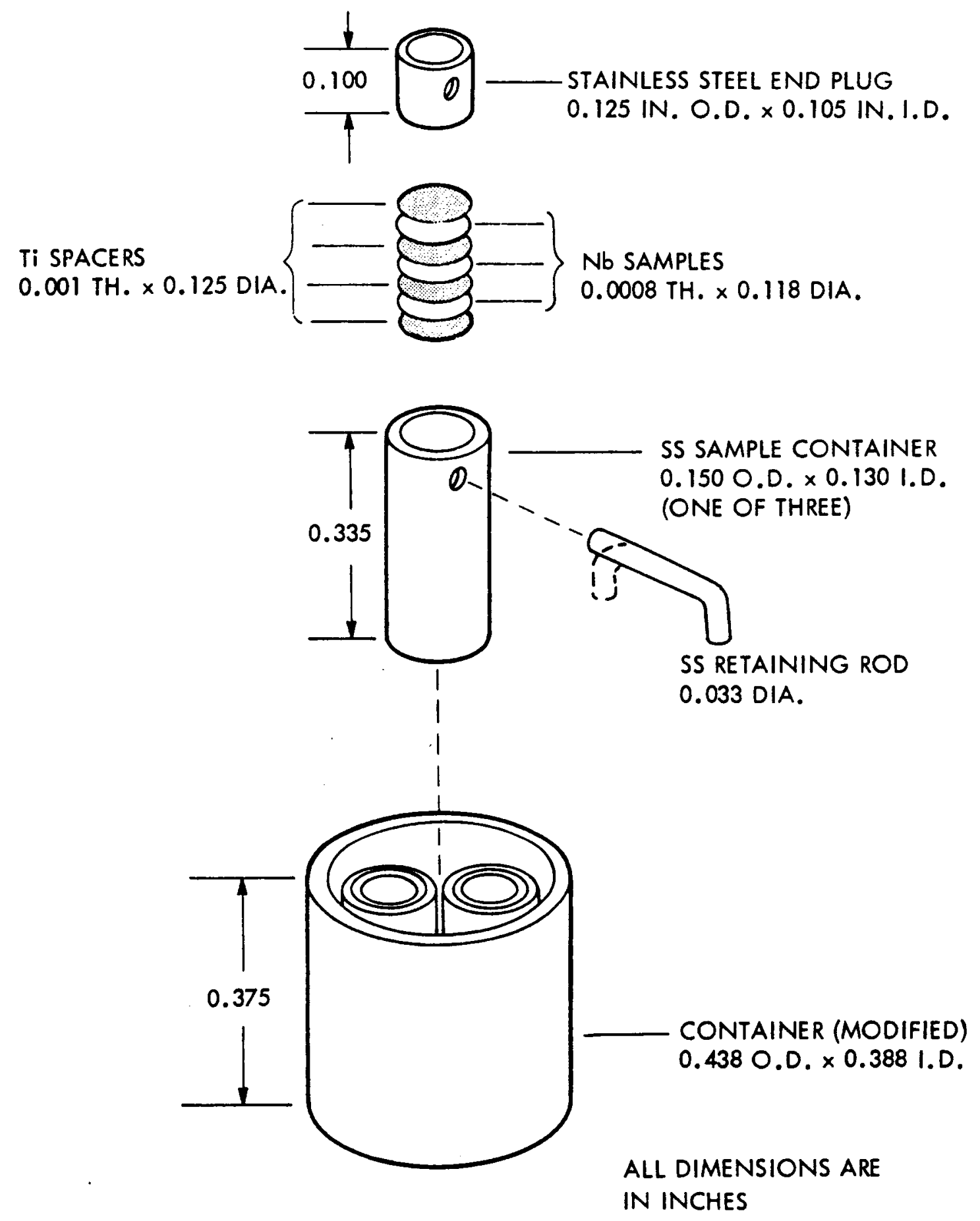

FIGURE $0-1$. Container and Loading for European Dosimeters. 
TABLE D-1

RUN 97, EUROPEAN DOSIMETER SET A - SPECIFIC DETAILS

Test: Run 97 - European Dosimeters

Page I of 2

HEDL Capsule Number

Container Identification

Set Identification: S-l. Hole 3

$R:=\quad 2: \underline{0} \mathrm{~cm}$

\begin{tabular}{|c|c|c|c|c|c|c|c|c|c|}
\hline \multirow[b]{2}{*}{ Material } & \multirow[b]{2}{*}{$\begin{array}{l}\text { Purchase Order } \\
\text { Number }\end{array}$} & \multirow[b]{2}{*}{$\begin{array}{c}\text { Batch } \\
\text { Number } \\
\text { Nen }\end{array}$} & \multirow[b]{2}{*}{ Description } & \multirow{2}{*}{\begin{tabular}{|c|c|} 
Material \\
we ight \\
(mag)
\end{tabular}} & \multicolumn{4}{|c|}{ Encapsulated Monitor Description } & \multirow[b]{2}{*}{ Subcontainer } \\
\hline & & & & & 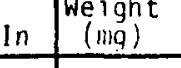 & 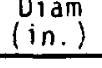 & $\begin{array}{l}\begin{array}{c}\text { Length } \\
\text { in.) }\end{array} \\
\end{array}$ & Ident & \\
\hline $\mathrm{Nb}^{*}$ & & Max Planok & $3.0 \mathrm{~mm} \times 0.020 \mathrm{~mm}$ & 0.6889 & & & & 1 & $1-A$ \\
\hline & & & & 0.6894 & & & & 2 & \\
\hline & & & & 0.7494 & & & & 3 & \\
\hline & & & & 0.7564 & & & & 4 & \\
\hline & & & & 0.7762 & & & & 5 & \\
\hline & & & & 0.7971 & & & & 6 & \\
\hline & & & & 0.7988 & & & & 7 & \\
\hline & & & & 0.8151 & & & & 8 & \\
\hline & & & & 0.8180 & & & & 9 & \\
\hline r & & 1 & 1 & 0.8237 & & & & 10 & 1 \\
\hline & & & & & & & & & \\
\hline & & & & & & & & & \\
\hline & & & & & & & & & \\
\hline & & & & & & & & & \\
\hline
\end{tabular}

*Foils loaded in same order with Ti spacers.

$U_{4}^{+10}$ 
TABLE D-1 (Continued)

Test: Run 97 - European Dosimeters HEDL Capsule Number

Container Identification A

Set Identification:

\begin{tabular}{|c|c|c|c|c|c|c|c|c|c|c|}
\hline \multirow[b]{2}{*}{ Material } & \multirow[b]{2}{*}{$\begin{array}{c}\text { Purchase Order } \\
\text { Number }\end{array}$} & \multirow[b]{2}{*}{$\begin{array}{l}\text { Batch } \\
\text { Number }\end{array}$} & \multirow[b]{2}{*}{ Description } & \multirow{2}{*}{$\begin{array}{c}\begin{array}{c}\text { Material } \\
\text { We ight } \\
\text { (mg) }\end{array} \\
\end{array}$} & \multicolumn{5}{|c|}{ Encapsulated Monitor Description } & \multirow[b]{2}{*}{ Subcontainer } \\
\hline & & & & & In & \begin{tabular}{|c} 
We ight \\
(mig)
\end{tabular} & Diam & $\begin{array}{l}\text { Lengtn } \\
\text { (in.) }\end{array}$ & Ident & \\
\hline $\mathrm{Nb} \mathrm{b}^{\star}$ & & & France & & & & & & $320-568$ & $2-A$ \\
\hline$N b^{*}$ & & & France & & & & & & $320-572$ & \\
\hline$N b^{\star}$ & & & France & & & & & & $320-576$ & \\
\hline $\mathrm{Cu}^{\star}$ & & ASARCO & $3.0 \mathrm{~mm} \times 0.28 \mathrm{~mm}$ & 14.777 & & & & & 47 & \\
\hline$T i^{\star}$ & $564.124 / 61 / 4897$ & $\begin{array}{l}\text { Baird } \\
\text { Atomic }\end{array}$ & $3.0 \mathrm{~mm} \times 0.28 \mathrm{~mm}$ & 8.728 & & & & & 57 & \\
\hline $\mathrm{Ni} \bullet$ & & & $3.0 \mathrm{~mm} \times 0.1 \mathrm{~mm}$ & 6.94 & & & & & 59 & \\
\hline $\mathrm{Fe}$ & $465784 / 74 / 6108$ & & $3.0 \mathrm{~mm} \times 0.1 \mathrm{~mm}$ & 5.860 & & & & & 55 & $f$ \\
\hline $\mathrm{Nb}$ & & $\begin{array}{l}\text { Bood Fellow } \\
\text { letals }\end{array}$ & $3.0 \mathrm{~mm} \times 0.0125 \mathrm{~mm}$ & 0.7474 & & & & & 37 & $3-A$ \\
\hline $\mathrm{Nb}$ & & Highways & $3.0 \mathrm{~mm} \times 0.0125 \mathrm{~mm}$ & 0.6215 & & & & & 40 & $3-A$ \\
\hline $\mathrm{Nb}$ & & Kawecki & $3.0 \mathrm{~mm} \times 0.0762 \mathrm{~mm}$ & 4.7385 & & & & & 45 & $3-A$ \\
\hline & & & & & & & & & & \\
\hline & & & & & & & & & & \\
\hline & & & & & & & & & & \\
\hline
\end{tabular}

*Foils loaded in same order with $\mathrm{ri}$ spacers.

$U_{+68}^{+55}$ 
TABLE D-2

RUN 97, EUROPEAN DOSIMETER SET B - SPECIFIC DETAILS

Test: Run 97 - European Dosimeters

HEDL Capsule Number

Container Identification B

Set Identification: S-2, Hole 3

rage 1 of 2

\begin{tabular}{|c|c|c|c|c|c|c|c|c|c|}
\hline \multirow[b]{2}{*}{ Material } & \multirow{2}{*}{$\begin{array}{l}\text { Purchase order } \\
\text { Number }\end{array}$} & \multirow[b]{2}{*}{$\begin{array}{l}\text { Batch } \\
\text { Nlumber }\end{array}$} & \multirow[b]{2}{*}{ Description } & \multirow{2}{*}{\begin{tabular}{|c|c|c|c|c|c|} 
Material \\
weignt \\
(ma) \\
\end{tabular}} & \multicolumn{4}{|c|}{ Encapsulated Monitor Description } & \multirow[b]{2}{*}{ Subcontainer } \\
\hline & & & & & 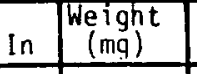 & $\begin{array}{l}\text { Dian } \\
\text { (in.) }\end{array}$ & $\begin{array}{l}\begin{array}{l}\text { engnth } \\
\text { in.) }\end{array} \\
\end{array}$ & Ident & \\
\hline $\mathrm{Nb}^{\star}$ & & & $3.0 \mathrm{~mm} \times 0.020 \mathrm{~mm}$ & 0.8882 & & & & 21 & $1-8$ \\
\hline & & & & 0.8897 & & & & 22 & \\
\hline & & & & 0.8997 & & & & 23 & \\
\hline & & & & 0.9024 & & & & 24 & \\
\hline & & & & 0.9027 & & & & 25 & \\
\hline & & & & 0.9032 & & & & 26 & \\
\hline & & & & 0.9089 & & & & 27 & \\
\hline & & & & 0.9339 & & & & 30 & \\
\hline & & & & 0.9350 & & & & 31 & \\
\hline 1 & & F & 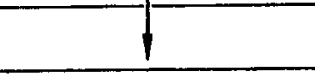 & 0.9367 & & & & 32 & t \\
\hline & & & & & & & & & \\
\hline & & & & & & & & & \\
\hline & & & & & & & & & \\
\hline & & & & & & & & & \\
\hline
\end{tabular}

*Foils loaded in same order with Ti spacers.

$U_{+21}^{+32}$ 
TABLE D-2 (Continued)

Test: _Run 97 - European Dos imeters HEDL Capsule Number Container Identification B

\begin{tabular}{|c|c|c|c|c|c|c|c|c|c|c|}
\hline \multirow[b]{2}{*}{ Material } & \multirow[b]{2}{*}{$\begin{array}{c}\text { Purchase Order } \\
\text { Number }\end{array}$} & \multirow[b]{2}{*}{$\begin{array}{l}\text { Batch } \\
\text { Nuniber }\end{array}$} & \multirow[b]{2}{*}{ Description } & \multirow{2}{*}{$\begin{array}{c}\text { Material } \\
\text { He ight } \\
\text { (mg) }\end{array}$} & \multicolumn{5}{|c|}{ Encapsulated Monitor Description } & \multirow[b]{2}{*}{ 'Subcontainer } \\
\hline & & & & & In & $\begin{array}{l}\text { Weight } \\
\text { (mq) }\end{array}$ & $\begin{array}{l}\text { Diam } \\
\text { (in.) }\end{array}$ & $\begin{array}{l}\text { Length } \\
\text { (in.) } \\
\end{array}$ & Ident & \\
\hline $\mathrm{Nb}$ & & France & & - & & & & & $320-567$ & $2-8$ \\
\hline $\mathrm{Nb}$ & & France & & & & & & & $320-571$ & 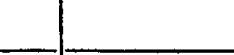 \\
\hline $\mathrm{Nb}$ & & France & & & & & & & $320-575$ & \\
\hline $\mathrm{Cu}$ & & ASARCO & $3.0 \mathrm{~mm} \times 0.28 \mathrm{~mm}$ & 14.898 & & & & & 48 & \\
\hline$T \mathbf{i}$ & $564.124 / 61 / 4897$ & $\begin{array}{l}\text { Baird } \\
\text { Atomic }\end{array}$ & $3.0 \mathrm{~mm} \times 0.28 \mathrm{~mm}$ & 8.768 & & & & & 52 & \\
\hline $\mathrm{Ni}$ & & & $3.0 \mathrm{~mm} \times 0.1 \mathrm{~mm}$ & 6.95 & & & & & 60 & \\
\hline $\mathrm{Fe}$ & $465784 / 746100$ & & $3.0 \mathrm{~mm} \times 0.1 \mathrm{~mm}$ & 5.860 & & & & & 56 & 1 \\
\hline No & & $\begin{array}{l}\text { Good Fellow } \\
\text { Metals }\end{array}$ & $3.0 \mathrm{~mm} \times 0.0125 \mathrm{~mm}$ & 0.7523 & & & & & 38 & $3-8$ \\
\hline Nb & & Highways & $3.0 \mathrm{~mm} \times 0.0125 \mathrm{~mm}$ & 0.6360 & & & & & 41 & $3-B$ \\
\hline Nb & & Kaweck i & $3.0 \mathrm{~mm} \times 0.0762 \mathrm{~mm}$ & 4.7467 & & & & & 46 & $3-B$ \\
\hline & & & & & & & & & & \\
\hline & & & & & & & & & & \\
\hline & & & & & & & & & & \\
\hline
\end{tabular}




\section{DISTRIBUTION}

$\underline{D O E} / \mathrm{RL}$ (2)

Manager

Chief Patent Attorney

DOE/FFTFPO (5)

Director

DOE/RRT HQ (2)

Program Division Director

$\underline{A I}$

P.0. Box 309, Canoga Park CA 91304

H. Farrar IV (2)

J. G. Bradley

\section{MACALESTER COLLEGE}

Department of Physics and Astronomy

St. Paul MN 55105

J. H. Roberts

HEDL (26)
R. W. Barker
W/A-106
B. L. Combs
W/A-39
R. Gold
J. J. Laidler
W/A-39
E. P. Lippincott
W/A-57
C. L. Long
W/A-39
W. N. McElroy
W/A-39
F. H. Ruddy
$W / A-39$
J. A. Ul seth (10)
W/A-39
H. H. Yoshikawa
$W / A-39$
W/A-62

Central Files (5)

Publications Services (2) 